\title{
Taxonomic revision and phylogeny of the Ophiocoma brevipes group (Echinodermata, Ophiuroidea), with description of a new subgenus (Breviturma) and a new species
}

\author{
Sabine STÖHR ${ }^{1}$, Emilie BOISSIN ${ }^{2} \&$ Thierry B. HOAREAU ${ }^{3}$ \\ ${ }^{1}$ Swedish Museum of Natural History, Department of Zoology, Box 50007, 10405 Stockholm, Sweden \\ Email: sabine.stohr@nrm.se (corresponding author) \\ ${ }^{2,3}$ Laboratoire ECOMAR, Université de La Réunion, BP 7151, 97715 Saint-Denis, La Réunion, France \\ ${ }^{2}$ USR3278-CRIOBE-CNRS-EPHE, Laboratoire d'Excellence « CORAIL », \\ Université de Perpignan-CBETM, 58 rue Paul Alduy, 66860 Perpignan Cedex \\ ${ }^{2}$ Email: eboissin@gmail.com \\ ${ }^{3}$ Molecular Ecology and Evolution Programme, Department of Genetics, University of Pretoria, \\ Private bag X20, Hatfield, Pretoria 0028, South Africa \\ ${ }^{3}$ Email: thoareau@gmail.com \\ ${ }^{1}$ urn:lsid:zoobank.org:author:412800EB-AACE-4313-9810-61F89B740405 \\ ${ }^{2}$ urn:lsid:zoobank.org:author:B35FF544-AE44-43B2-B1CA-3769A281F386 \\ ${ }^{3}$ urn:1sid:zoobank.org:author:44578720-231E-4D99-8F38-2A94B7C4E4F2
}

\begin{abstract}
The taxonomy of the genus Ophiocoma was last revised by Devaney in 1970. Recent discoveries of new species and re-instatement of previously synonymized names suggest that we still do not fully understand the species limits in this genus. A recent biodiversity survey of the SW Indian Ocean shallow reefs strongly suggested an unrecognised species in the genus, closely related to O. brevipes/O. dentata. This study examined both the molecular phylogenetic relationships and the morphological characteristics of several species in the genus in order to characterise the unrecognised species. The focal species clusters with $O$. brevipes, $O$. dentata, $O$. doederleini within a monophyletic clade supported by molecular data for the first time. The name Breviturma subgen. nov. is proposed for this clade, previously known as brevipes group. Type material of nominal species that have been synonymized with $O$. dentata was examined and re-assessed. Ophiocoma marmorata proved not conspecific with $O$. dentata. A rarely used character, dorsal disc granule density, was tested and showed differences between the examined species at similar sizes. In combination with colour pattern, disc granule density, arm spine sequence and maximum disc size, the new species was delimited morphologically and described as Ophiocoma krohi sp. nov.
\end{abstract}

Key words. morphology, Ophiocoma krohi, Breviturma, mitochondrial DNA, cryptic biodiversity.

Stöhr S., Boissin E. \& Hoareau T.B. 2013. Taxonomic revision and phylogeny of the Ophiocoma brevipes group (Echinodermata, Ophiuroidea), with description of a new subgenus (Breviturma) and a new species. European Journal of Taxonomy 68: 1-26. http://dx.doi.org/10.5852/ejt.2013.68 


\section{Introduction}

The history of the taxonomy of the genus Ophiocoma L. Agassiz, 1835 is convoluted and confused. Devaney (1970) resolved some of the confusion using external and internal characters such as spine sequences along the arm, colour pattern, and the shape of the dental plate, to delimit four groups, namely the brevipes, scolopendrina and pumila groups earlier proposed by Clark (1921), and the pica group (Devaney 1970). After 1970, few new species of Ophiocoma have been described, namely O. paucigranulata Devaney, 1974 from the Caribbean and O. aegyptiaca Soliman, 1991 from Egypt. Also, O. endeani Rowe \& Pawson, 1977 was proposed as a replacement name for O. alternans Endean, 1964 (a homonym) by Rowe \& Pawson (1977). Ophiocoma similanensis Bussarawit \& Rowe, 1985 was synonymized with $O$. erinaceus Müller \& Troschel, 1842 by Price \& Rowe (1996). Recently a new species $O$. cynthiae Benavides-Serrato \& O'Hara, 2008 was described and $O$. schoenleini Müller $\&$ Troschel, 1842 was reinstated as a valid species (Benavides-Serrato \& O'Hara 2008). Both are close to $O$. erinaceus and have previously been mistaken for it, as was suggested by an earlier molecular study (O'Hara et al. 2004). Although Ophiocoma is a well studied genus, consisting of comparatively large, conspicuous, abundant and easily accessible tropical shallow water species, it clearly still holds unrecognized diversity. Currently, 22 species are accepted in the genus (Stöhr \& O'Hara 2013).

In a recent large-scale marine biodiversity survey in the Southwestern Indian Ocean (BIOTAS), we identified several specimens as belonging to the brevipes group based on morphology. According to Devaney (1970), the brevipes group currently includes the three species Ophiocoma brevipes Peters, 1851, O. dentata Müller \& Troschel, 1842 and O. doederleini de Loriol, 1899. In our material, we found specimens belonging to these three species and a fourth unknown species was suspected and confirmed by subsequent molecular analysis. They formed a distinct genetic lineage when compared to other available species of Ophiocoma (Hoareau et al. 2013). Currently (Devaney 1970; Stöhr \& O'Hara 2013), the following nominal species are regarded as synonyms of the species in the brevipes group: O. brevispinosa Smith, $1876=$ O. brevipes; O. insularia Lyman, 1862, O. marmorata MarktannerTurneretscher, 1887, O. ternispina v. Martens, 1870 and O. variegata Smith, $1876=$ O. dentata . The name Ophiopeza danbyi Farquhar, 1897 was included in the synonymy of $O$. brevipes by Clark (1915), but not by Devaney (1970). The newly recognized lineage may either belong to a new, not yet described species, or it may correspond to one of the synonymized species.

Specimens of the brevipes group are characterized by similar numbers of arm spines on each side of the arm segments beyond the disc, four to six arm spines on each side of proximal arm segments, a dental plate that is roughly twice as long as wide, with wide septum dividing the tooth foramina, broadly oval dorsal arm plates, densely packed, small and spherical disc granules that cover the entire ventral interradius, and two tentacle scales along most of the arm (Devaney 1970). According to Devaney (1970), the comparatively short dental plate with small region for the tooth papillae is an important character to distinguish the brevipes group from the other subgroups of Ophiocoma. Moreover, Benavides-Serrato \& O'Hara (2008) found that subtle differences in characters such as the extent of granulation on the ventral disc, tube foot colour and shape of the dental plate had previously been overlooked or misinterpreted as intraspecific variation in species of the scolopendrina group. These criteria might be informative to characterise the specimens of the newly found lineage in the brevipes group.

This study aims to describe that unidentified species in the brevipes group, using both molecular methods and morphological characters. Using two mitochondrial loci, we constructed a phylogeny to assess the molecular status of the brevipes group relative to the scolopendrina group and to evaluate the species relationships within the brevipes group. In addition, we identified a new character, dorsal disc granule density, to distinguish between the species in the brevipes group and re-evaluate type material of the studied species. 


\section{Material and methods}

Brittle stars of the genus Ophiocoma were collected at La Réunion Island and off Madagascar (NosyBe Island). Specimens of $O$. doederleini from La Réunion and Polynesia were provided by G. Paulay from the collections of the Florida Museum of Natural History. Total genomic DNA was extracted from a piece of arm following the DNeasy protocol (Qiagen). COI sequences were already available for most of the species (Hoareau et al. 2013; Supplementary file: KC759738-KC759923) except for $O$. dentata and $O$. doederleini. A portion of the COI barcoding gene and of the $16 \mathrm{~S}$ ribosomal gene were amplified using echinoderm-specific hybrid primers (Hoareau \& Boissin 2010) and universal primers (Palumbi 1996), respectively. We followed the PCR conditions described in these studies. The amplicons were then sequenced using BigDyeTerminator (Applied Biosystems, Foster City, CA, USA) cycle sequencing reactions, and electrophoresed on an ABI 3730xl DNA Analyzer (Applied Biosystems) at the Interdisciplinary Center for Biotechnology Research (University of Florida). We aligned the sequences using mafft 6 online (http://mafft.cbrc.jp/alignment/server/) with all the default settings. All the sequences are available in GenBank (see Supplementary file). From the concatenated datasets, a Neighbour Joining phylogenetic tree was reconstructed using Mega5.05 (Tamura et al. 2011) applying 1000 bootstraps and the Kimura-2-Parameters model of substitution. Additionally, we used maximum likelihood inference using Mega. Because we used two mitochondrial loci, we estimated a single substitution model for the concatenated dataset using Mega (GTR + gamma + invariant site model). Support for each node was assessed with 200 bootstrap replicates. To overcome potential complications introduced by alignment gaps, we applied the Partial-Deletion option available in Mega in both reconstruction methods. Sequences of Ophiocoma erinaceus and O. scolopendrina (Lamarck, 1816) from the scolopendrina group were used as outgroup.

After DNA extraction, some of the individuals were used for morphological examination. Since the new species is smaller than the others, we selected comparative material from other species at similar size (11-13 mm disc diameter), depending on availability. Two of the largest specimens of Ophiocoma sp. nov. at $11 \mathrm{~mm}$ and $13 \mathrm{~mm}$ disc diameter (dd) and a specimen each of O. doederleini (13 $\mathrm{mm} \mathrm{dd}$ ), $O$. brevipes $(13 \mathrm{~mm} \mathrm{dd})$ and $O$. dentata $(11 \mathrm{~mm} \mathrm{dd})$ were selected for scanning electron microscopy (SEM). These specimens were first photographed with a digital camera. Then they were treated with diluted (1:1) household bleach $(\mathrm{NaOCl})$ to remove the epidermis. They were mounted on aluminium stubs with spray glue, dorsal side up, and examined in a Hitachi FE-S4300 scanning electron microscope. Then the glue was dissolved with butyl acetate and the animals were remounted, ventral side up, and examined by SEM again. Finally, the specimens of $O$. doederleini (UF5318), O. brevipes (SMNH133230), O. dentata (URUN2009-11047) and the $13 \mathrm{~mm}$ dd Ophiocoma sp. nov. (SMNH-Type 8535) were disarticulated in concentrated bleach. The skeletal elements were washed in tap water, mounted on stubs and examined by SEM.

Whole animals were measured using a digital calliper, smaller parts were measured on the SEM images using the software ImageJ v.1.47c (Rasband 1997-2012). Disc diameter is defined as the distance from the distal edge of a pair of radial shields (or if concealed the disc edge atop the arm) to the edge of the opposite interradius. Densities of disc granules were measured by overlaying a square frame of $0.5 \mathrm{~mm}$ side length $\left(0.25 \mathrm{~mm}^{2}\right)$ to the image in ImageJ and counting all complete granules inside the square. This was repeated at least three times in different places of the image, but always in the central part of the disc to avoid the often bulging, more sparsely granulated disc periphery. The numbers were multiplied by four to give densities for $1 \mathrm{~mm}^{2}$ for easier comparability with previously published values. Vertebrae were measured in ImageJ across their greatest width and height; oral plates (jaws) were measured by fitting an ellipse around their irregular shape and calculating the major (here height) and minor (here width) axes. Arm spines were counted on 2-5 arms of each specimen and when the numbers at a segment varied between arms the less common number is given in parentheses. The taxonomy follows Stöhr \& 
O'Hara (2013). The morphological terminology follows Stöhr et al. (2012). Field numbers, museum registration numbers and GenBank accession numbers are listed in the Supplementary file.

\section{Abbreviations}

\section{Acronyms of institutions and repositories}

$\begin{array}{ll}\text { NHMW } & =\text { Natural History Museum Wien (Vienna) } \\ \text { SMNH } & \text { Swedish Museum of Natural History } \\ \text { UF, FLMNH } & =\text { Florida Museum of Natural History } \\ \text { URUN } & =\text { Université de La Reunion } \\ \text { ZMB } & =\text { Zoological Museum Berlin }\end{array}$

\section{Other abbreviations}

$\mathrm{DAP}=$ dorsal arm plate

$\mathrm{dd}=$ disc diameter

$\mathrm{ML}=$ maximum likelihood

$\mathrm{NJ} \quad=$ neighbour joining

$\operatorname{spm}(\mathrm{s})=\operatorname{specimen}(\mathrm{s})$

$\mathrm{W}: \mathrm{L}=$ width:length

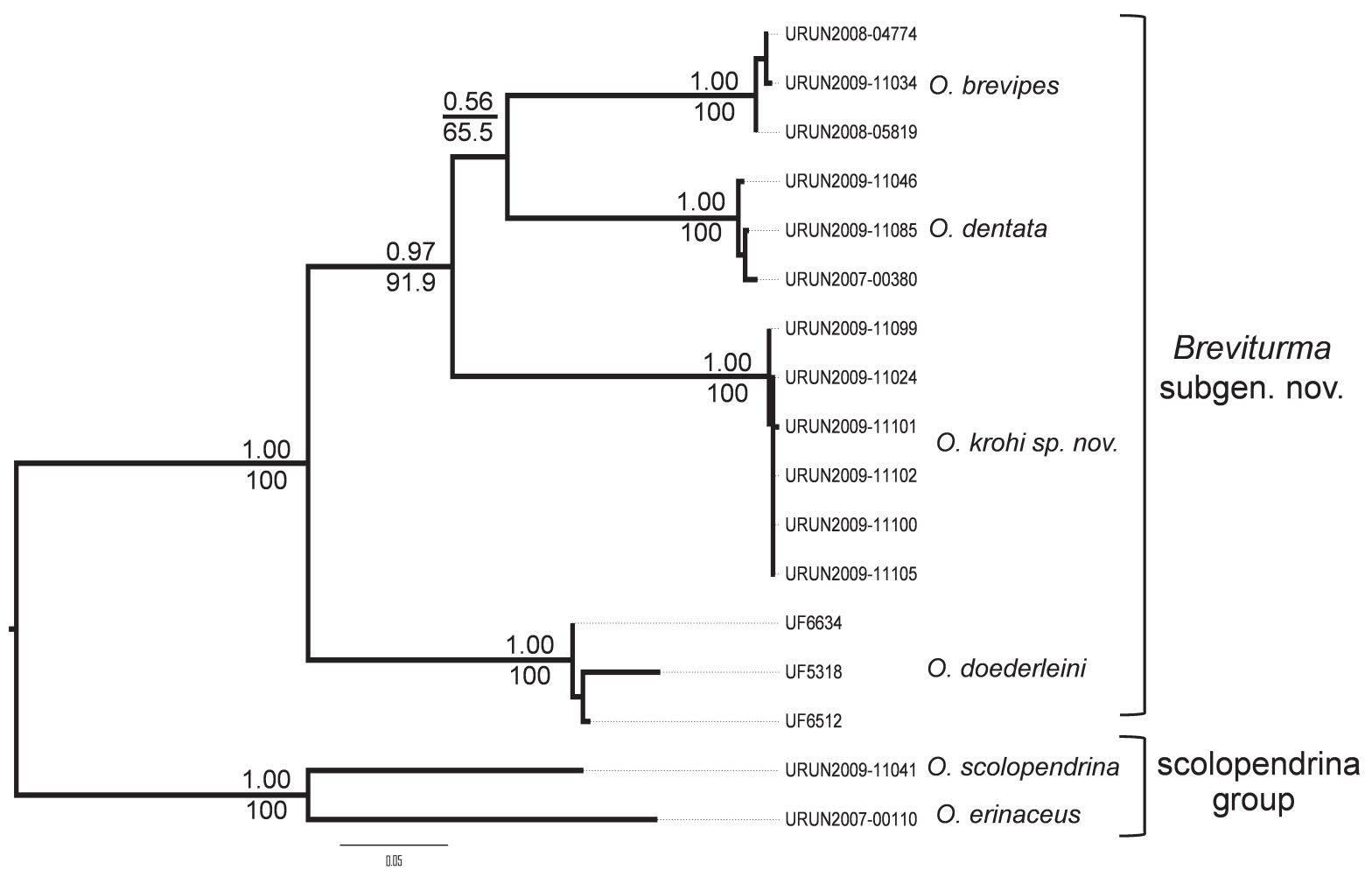

Fig. 1. Phylogenetic reconstruction of Ophiocoma (Breviturma) subgen. nov. (two species of the scolopendrina group as outgroup), estimated by maximum likelihood and neighbour-joining analysis of concatenated sequences of the mitochondrial cytochrome $c$ oxidase subunit I and rDNA 16S (in total $1125 \mathrm{bp}$ ). Values at branches indicate bootstrap values for maximum likelihood (above branches) and neighbour-joining (below branches). Specimen codes are resolved in the Supplementary file. 


\section{Results}

The concatenated sequences of the 17 specimens contained 1125 sites (1069-1119 bp) of which 303 $(26.9 \%)$ were parsimony informative. For most of the specimens the complete target sequence was obtained. For the specimens of $O$. doederleini, $614 \mathrm{bp}$ were recovered from the original $659 \mathrm{bp}$. The $16 \mathrm{~S}$ sequences varied depending on the presence of multiple indels (450-456 bp). For the COI locus, no indels, distinct double pics or stop codons were detected which rule out the presence of pseudogenes. Only three amino-acids showed variation in the COI sequences; one site involved a change from Serine to Alanine in all the specimens of $O$. krohi sp. nov.

Most of the nodes in the phylogeny received a good bootstrap support (ML $>95$ and NJ $>90$ ). The ML and NJ phylogenetic trees were congruent and confirmed, for the first time, a molecular basis for the clustering of species from the brevipes group (100/100 for ML and NJ respectively), relative to the two species belonging to the scolopendrina group. The trees also revealed four highly supported taxa (100/100) within the brevipes group, matching the species $O$. brevipes, O. dentata, O. doederleini and a new species (Fig. 1). Ophiocoma doederleini had a deeper branching in the tree relative to the three other species. The clade consisting of Ophiocoma sp. nov., O. brevipes and $O$. dentata was supported (bootstrap values 97/91.9). However, the relationships within the clade were unclear since the clade consisting of $O$. brevipes and $O$. dentata was weakly supported (56/65.5).

Comparison with the type material confirmed our identifications of $O$. brevipes, O. dentata and O. doederleini. The morphological examinations also supported the existence of a fourth species in the brevipes group. The most important characters were dorsal disc granule density and arm spine numbers, summarized in Table 1. Colour pattern was also an important species delimiting criterion (Fig. 2). Finally, the specimens of Ophiocoma krohi sp. nov. were on average significantly smaller than the three other species of the brevipes group (Kruskal-Wallis test, $K=9.2514$, d.f. $=3, P=0.0261$ ). This result confirms previous observations based on examinations of specimens in the collections of FLMNH from a large geographic range in the Pacific and Indian Oceans (Okinawa, Tuamotu Islands, Mariana Islands, Mascarene Islands, Samoa Islands, Society Islands, Niue Island, Hawaiian Islands).

The brevipes group is well supported as a monophyletic group and below proposed as a subgenus of Ophiocoma. The fourth species is described below as new.

Class Ophiuroidea Gray, 1840

Order Ophiurida Müller \& Troschel, 1840

Family Ophiocomidae Ljungman, 1867

Genus Ophiocoma L. Agassiz, 1835

\section{Diagnosis}

Typically large, conspicuous, shallow water tropical brittle stars. Dorsal disc completely covered with granules, extending onto the ventral side to varying degree. Strong, erect, smooth and generally solid (except $O$. pusilla Brock, 1888) arm spines. A cluster of tooth papillae on the lower part of the dental plate; large rectangular teeth. Adoral shields confined to lateral edges of oral shield, not meeting. Abradial muscle surface of oral plates well developed with a series of horizontal grooves and ridges. Lateral arm plates short, curved, with ventral notch for tentacle foot, spine articulations sigmoidal. 
Table 1. Morphometric data for the species of Ophiocoma (Breviturma) subgen. nov. Type material from Zoological Museum Berlin (ZMB), Swedish Museum of Natural History (SMNH) and Natural History Museum Vienna (NHMW). Specimen NHMW 104.66a is regarded as the holotype of O. marmorata, the other specimens of that lot most likely belong to a different species. DD, disc diameter, MNHN, Muséum national d'Histoire naturelle (Paris), UF, Florida Museum of Natural History, URUN, University of La Réunion.

\begin{tabular}{|c|c|c|c|c|c|}
\hline Species & Specimen & $\mathrm{DD}[\mathrm{mm}]$ & $\begin{array}{c}\text { Granules } \\
\mathbf{m m}^{-2}\end{array}$ & $\begin{array}{c}5 \text { spines at } \\
\text { segments }\end{array}$ & $\begin{array}{l}4 \text { spines beyond } \\
\text { segment } 15\end{array}$ \\
\hline \multirow[t]{10}{*}{ Ophiocoma brevipes } & ZMB Ech 961 & $12-13$ & - & $5-9$ & yes \\
\hline & ZMB Ech 962 & 13 & - & $3-14$ & yes \\
\hline & ZMB Ech 963 & 13 & - & $5-11$ & yes \\
\hline & ZMB Ech 4660 & $17-18$ & - & $6-12$ & yes \\
\hline & ZMB Ech 4660 & 15 & - & $6-11$ & yes \\
\hline & ZMB Ech 4660 & 12 & - & $6-17$ & yes \\
\hline & SMNH-133230 & 13 & $240-280$ & $6-10$ & yes \\
\hline & URUN 2013-11188 & 19.7 & $176-188$ & $6-18$ & yes \\
\hline & URUN 2008-04774 & 13.1 & $148-168$ & $6-10$ & yes \\
\hline & URUN 2008-05819 & 13.5 & $180-200$ & $6-11$ & yes \\
\hline \multirow[t]{4}{*}{ Ophiocoma dentata } & ZMB Ech 931 & $18-19$ & - & none & no \\
\hline & URUN 2009-11047 & 11 & $216-244$ & none & no \\
\hline & SMNH-133232 & 23.7 & $44-64$ & $(8,9)$ & no \\
\hline & URUN 2007-00380 & 24 & $52-68$ & none & no \\
\hline Ophiocoma ternispina & ZMB Ech 1815 & 16 & - & none & no \\
\hline Ophiocoma insularia & SMNH-Type-5244 & 17.6 & $96-120$ & $7-9$ & yes \\
\hline \multirow[t]{4}{*}{ Ophiocoma marmorata } & NHMW 10.466a & 8.7 & 80 & none & yes \\
\hline & NHMW 10.466b & 6.3 & 60 & none & yes \\
\hline & NHMW $10.466 \mathrm{c}$ & 6.1 & 48 & none & yes \\
\hline & NHMW 10.466d & 5.2 & 60 & $6-7$ & yes \\
\hline \multirow[t]{12}{*}{ Ophiocoma krohi sp. nov. } & SMNH-Type-8531 & 6.8 & 152 & $6-7$ & no \\
\hline & MNHN-IE-4303 & 9 & 125 & none & no \\
\hline & SMNH-Type-8532 & 9.6 & $116-120$ & none & no \\
\hline & MNHN-Type-8533 & 10 & $140-164$ & $6-9$ & yes \\
\hline & UF-13938 & 10.3 & $112-120$ & $7-9$ & no \\
\hline & SMNH-Type-8534 & 11 & $132-136$ & $6-7$ & yes \\
\hline & URUN 2009-11108 & 11.3 & $112-120$ & none & no \\
\hline & MNHN-IE-4301 & 11.8 & $72-96$ & $5-8$ & yes \\
\hline & MNHN-IE-4300 & 12.7 & 104 & none & no \\
\hline & SMNH-Type-8535 & 13 & 104 & $6-7$ & yes \\
\hline & MNHN-IE-4302 & 13 & $96-104$ & none & no \\
\hline & SMNH-Type-8536 & 15.5 & $64-96$ & none & yes \\
\hline \multirow[t]{3}{*}{ Ophiocoma doederleini } & UF 5318 & 13 & $200-224$ & $6-7$ & yes \\
\hline & UF 6512 & 17 & $88-96$ & $6-9$ & yes \\
\hline & UF 6634 & 26 & $60-64$ & $6-10$ & yes \\
\hline
\end{tabular}




\section{Subgenus Breviturma subgen. nov. urn:Isid:zoobank.org:act:DA2BAD13-0E03-45B3-9D7A-78796329BC3C}

\section{Diagnosis}

Subgenus of Ophiocoma, previously known as brevipes group. Characterized by equal (non-alternating) numbers of arm spines; disc granules uniform in size, low and blunt; dorsal arm plates rounded, almost oval (in adult specimens, more rounded triangular in younger individuals); dental plate about twice as long as wide and thus the shortest in the genus, with tooth papillae region occupying $25 \%$ of the dental plate length.

\section{Etymology}

The name Breviturma refers to the original name brevipes group, combined with turma $=$ troop, here meaning group.

\section{Type species}

Ophiocoma brevipes Peters, 1851.

\section{Included species}

Ophiocoma doederleini, O. brevipes, O. dentata and O. krohi sp. nov.

Ophiocoma (Breviturma) doederleini de Loriol, 1899

Figs 2A-C, 3

Ophiocoma doederleini de Loriol, 1899: 30, pl. 3 fig. 2.

\section{Diagnosis}

Species of Ophiocoma (Breviturma) subgen. nov. characterized by strongly dark and light banded (annulated) arm spines; slender arm spines, the longest up to three segments long; up to five arm spines on few segments from segment 6; variable in dorsal disc colour pattern (reticulating or spotted); density of dorsal disc granules reaches more than $200 \mathrm{~mm}^{-2}$. Maximum size at least $26 \mathrm{~mm}$ according to the studied material.

\section{Material examined}

\section{UF5318}

Collecting Data. Polynesia, Moorea, $1^{\circ} 28^{\prime} 55^{\prime \prime}$ S, 149 50'32" W, fore reef between Cook's and Opunohu Bays, outer reef slope, under rocks, partly in sand, 10-15 m, by hand, collectors C. McKeon, C. Meyer, G. Paulay, 19 Jul. 2006, 1 spm.

Morphological Data. $13 \mathrm{~mm}$ dd. Granule density on light image $180-200 \mathrm{~mm}^{-2}$. Arm spine sequence $3,3,3,3,4,5,5,4,4,4,4,4,4,4,4,4,4,4,4,4,4,4,4,4,4,4,4,4,4,3,3$. Dorsal disc dark brown with irregular white patches, with a net-like reticulating darker brown pattern (Fig. 2A, B). Dorsal arms dark brown with widely spaced white bands with brown spots. Ventral disc white with brown spots. Oral frame white, oral shields with brown spots. Ventral arms with long white and brown bands, brown spots on the white bands. Arm spines strongly annulated with white and brown rings, long and slender, dorsalmost spine barely two segments long, middle spines longer than two segments.

Dorsal disc granule diameter 47-51 $\mu \mathrm{m}$, granule density $224 \mathrm{~mm}^{-2}$ (Fig. 3A, C). Third spine on proximal segments $2.3 \mathrm{~mm}$ long; proximal DAPs $1.74-1.8 \mathrm{~mm}$ wide (Fig. 3B). Oral shields slightly wider than long; 5-6 tooth papillae, 5 lateral oral papillae at each jaw edge, outer papilla widest (Fig. 3D). Dental plate 


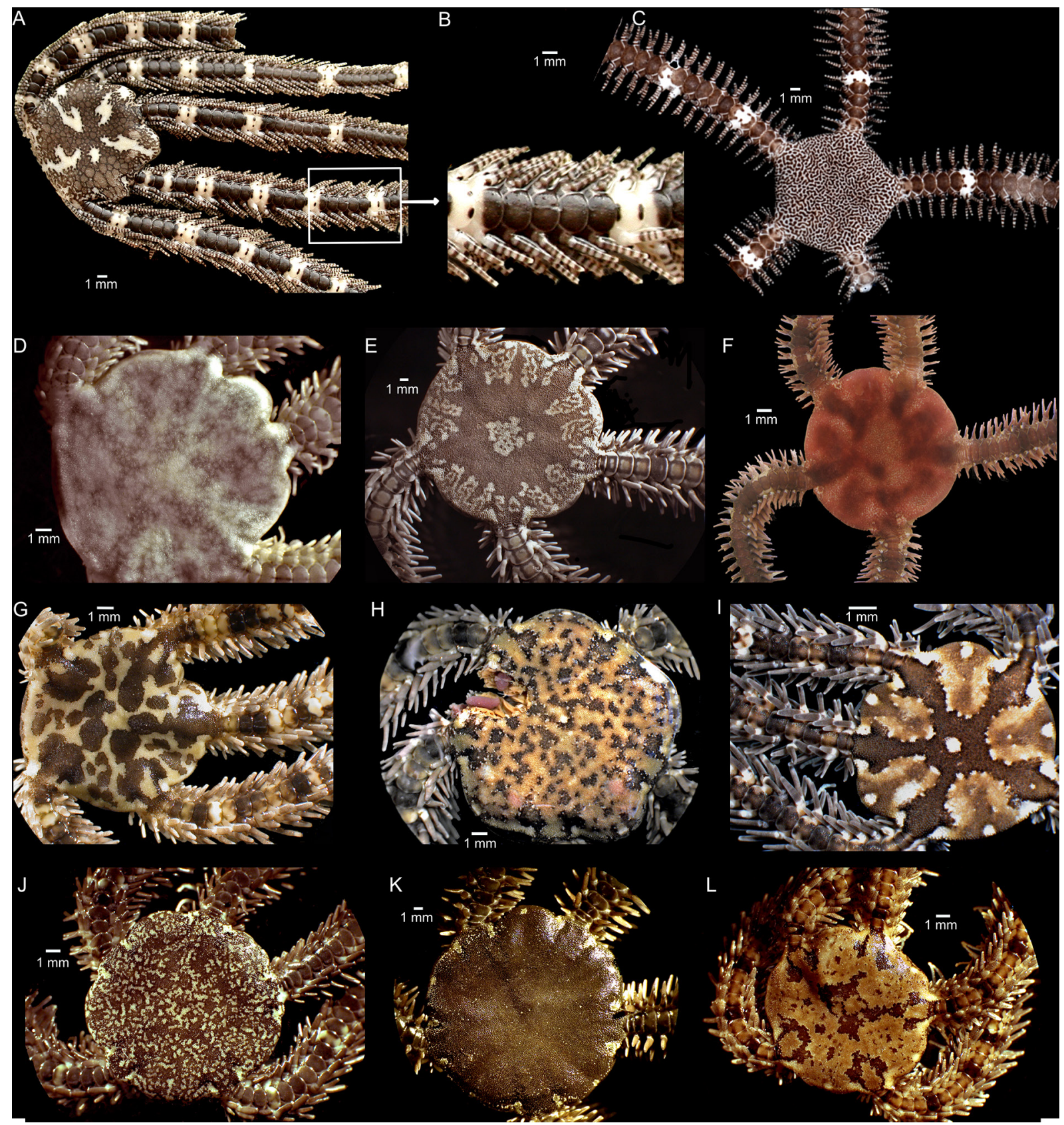

Fig. 2. Colour variations in species of Ophiocoma (Breviturma) subgen. nov. A-C. Ophiocoma doederleini Rowe \& Pawson, 1977. A. Reticulated specimen (UF5318). B. Close-up of annulated arm spines. C. Densely reticulated specimen (UF6518). D. Ophiocoma brevipes Peters, 1851 (SMNH133230). E-F. Ophiocoma dentata Müller \& Troschel, 1842. E. Variegated specimen (SMNH-133232). F. Uniformly reddish brown specimen (URUN2009-11047). G-L. Ophiocoma krohi sp. nov. paratypes. G. With large patches (SMNH-Type-8533). H. Small irregular spots (MNHN-IE-4302). I. Star-shaped radiating pattern (SMNH-Type-8531). J. Densely mottled (MNHN-IE-4301). K. Uniformly dark brown (SMNH-Type-8536). L. Light brown, sparsely mottled (UF13938). For institution codes see main text. 
short, externally with two large tooth foramina in dorsal half, dorsalmost foramen oval, wider than long, other foramen square (Fig. 3H); internally each foramen divided by a wide septum, dorsalmost one into two small holes, other foramen into two parallel slits (lower septum broken in Fig. 3H2). External ventral half of dental plate with smooth horizontal ridges, forming two wide ovals, the ventralmost one half the size of the other; a cluster of tiny depressions at ventral end of plate. Radial plate shaped as a wide triangle, proximal edge concave, distal point with condyle, depressed at lateral edge (Fig. 3F), but not as ball-like as in $O$. brevipes and $O$. krohi sp. nov. Adradial genital plate long, flat, thin, thickened at distal end, with condyle and pit (Fig. 3I1); abradial genital plate thin, blade-like, curved distally (Fig. 3I2). Oral plates,

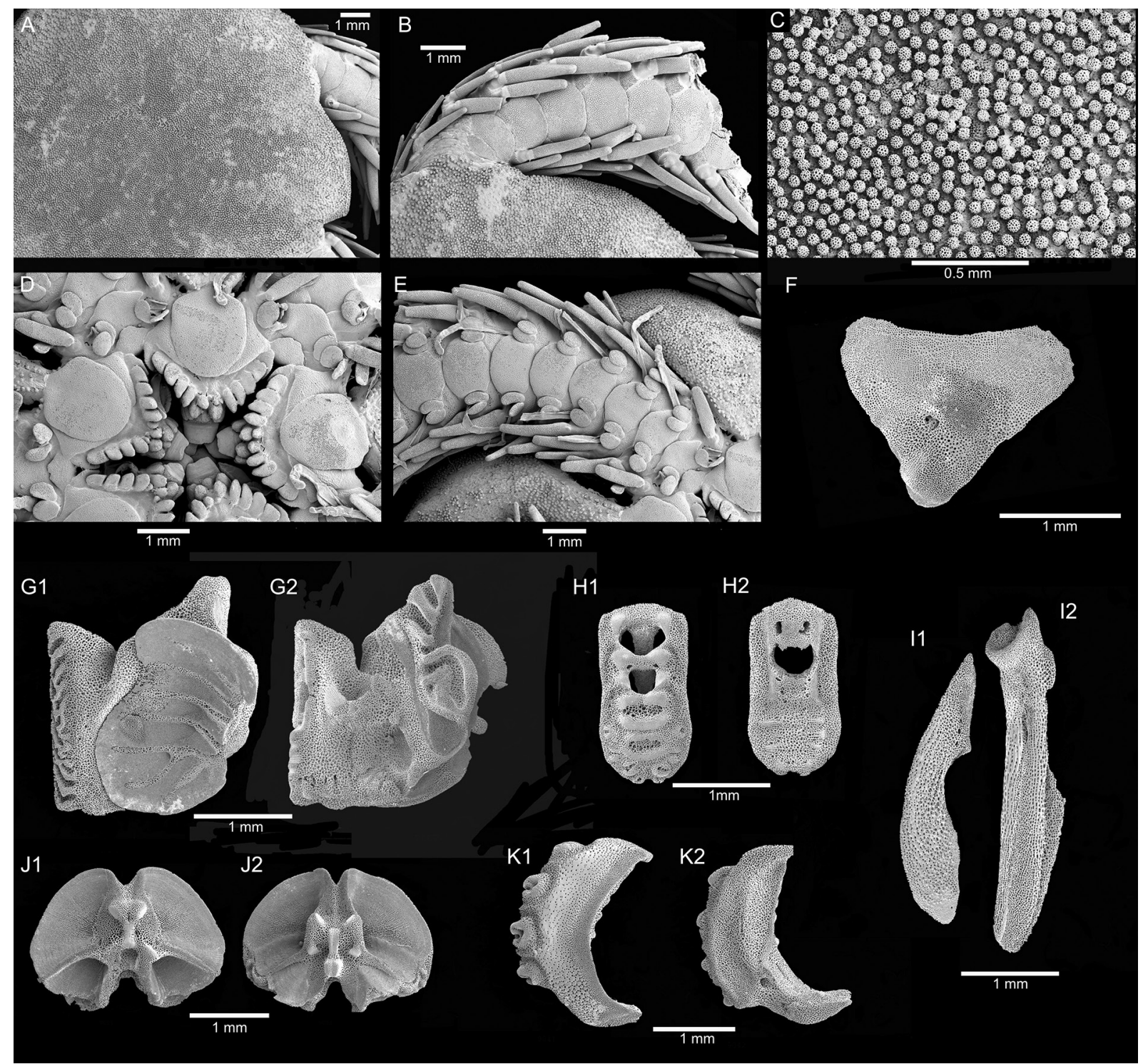

Fig. 3. Ophiocoma (Breviturma subgen. nov.) doederleini de Loriol, 1899 (UF5318). SEM images. A. Dorsal disc. B. Dorsal arm. C. Dorsal disc granules. D. Jaws. E. Ventral arm. F. Radial shield, internal aspect, distal edge downwards. G. Oral plates (half-jaws), abradial aspect left, adradial right, proximal edge left. H. Dental plates, external aspect on left, internal on right (lower septum broken), dorsal upwards. I. Genital plates abradial left, adradial right, distal upwards. J. Arm vertebrae, proximal aspect left, distal right, dorsal upwards. K. Lateral arm plates, external aspect left, internal right, distal to the right. UF, Florida Museum of Natural History. 
adradial distal part with strong folds, abradial face with large muscle flange with horizontal striations, $2.5 \mathrm{~mm}$ wide, $2.0 \mathrm{~mm}$ high (Fig. 3G). Proximal vertebrae are $2.4 \mathrm{~mm}$ wide and $1.8 \mathrm{~mm}$ high (Fig. 3J).

\section{UF6512}

Collecting Data. La Réunion Island, Saint Gilles, Point des Aigrettes, $2^{\circ} 02^{\prime} 11^{\prime \prime} \mathrm{S}, 55^{\circ} 13^{\prime} 01^{\prime}$ E, fore reef, under rocks, 6-22 m dive, collectors H. Bruggemann, N. Hubert, F. Michonneau, G. Paulay, 12 Aug. 2007, 1 spm.

Morphological data. $17 \mathrm{~mm}$ dd. Granule density 88-96 $\mathrm{mm}^{-2}$. Arm spine sequence 3, 3, 3, 4, 4, 5, 5, 5, $5,4,4,4,4,4,4,4,4,4,4,4,4$, continued until the distal arm where the spines decrease to three. Brown dorsal disc with reticulating net pattern. Dorsal arms dark brown with widely spaced short white bands with dark markings. Ventral disc dark and light brown mottled. Oral frame white with few dark spots. Ventral arms dark brown with few short white bands. Spines white, strongly annulated with brown rings, long and slender, dorsalmost spine barely two segments long, middle spines longer than two segments.

\section{UF 6634}

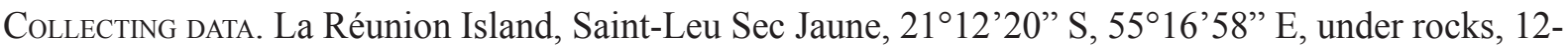
15 m dive, collectors H. Bruggemann, N. Hubert, F. Michonneau, G. Paulay, 17 Aug 2007, 1 spm.

Morphological Data. $26 \mathrm{~mm}$ dd. Granule density 60-64 $\mathrm{mm}^{-2}$. Arm spine sequence 3, 3, 3, 4, 4, 5, 5, 5, $5,5,4,4,4,4,4,4,4,4,4,4,4 \ldots . .3$. Dorsal disc medium brown with dark mesh pattern (reticulated), dorsal arms dark brown with widely spaced lighter brown bands. Oral frame creamy white with brown spots and irregular markings, ventral disc light brown with dark reticulations, ventral arm medium brown with widely spaced cream markings. Spines white, strongly annulated with brown rings, long and slender, dorsalmost spine barely two segments long, longest middle spine almost as long as three segments.

\section{UF6518 (only image available)}

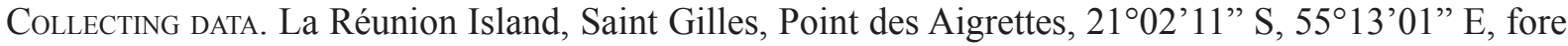
reef, under rocks, 6-22 m dive, collectors H. Bruggemann, N. Hubert, F. Michonneau, G. Paulay, 12 Aug. 2007, 1 spm.

Morphological DATA. $12.5 \mathrm{~mm}$ dd. Colour pattern on dorsal disc consists of thicker, brown, reticulating lines, instead of a thin net-like pattern as in the other specimens; otherwise similar to the others (Fig. 2C).

\section{Remarks}

Ophiocoma doederleini occurs in several colour morphs, with various reticulated patterns (Fig. 2A, C) or spotted disc (Devaney 1970), which is confirmed by our molecular data (Boissin \& Hoareau, unpublished). It can easily be distinguished from the other species of Breviturma subgen. nov. by its dark and light strongly annulated, more slender, and longer arm spines. It also has the widest dorsal arm plate of all species of Breviturma subgen. nov. The high granule density in the smallest specimen was surprising and may need further investigation. Maximum size at least $26 \mathrm{~mm}$ according to the studied material.

\section{Ophiocoma (Breviturma) brevipes Peters, 1851}

Figs 2D, 4A-E, 5A, D, G, J, M, P

Ophiocoma brevipes Peters, 1851: 466.

Ophiocoma brevispinosa Smith, 1876: 40.

Ophiopeza danbyi Farquhar, 1897: 189.

non Ophiocoma brevipes - Stöhr et al. 2008: 553. 


\section{Diagnosis}

Species of Ophiocoma (Breviturma) subgen. nov. characterized by a light colour pattern, cream to white with some brown to grey markings; five arm spines from segment 4 or 5 on large specimens for more than 10 segments; disc granule densities up to about $250 \mathrm{~mm}^{-2}$. Maximum size at least $20 \mathrm{~mm} \mathrm{dd}$ according to the examined material.

\section{Type material examined}

\section{Syntype ZMB Ech 961}

Collecting data. Mozambique, $18^{\circ} 39.942$ ' S 35 31.774’ E (from atlas), collector Peters, 1851.

Morphological Data. 12-13 mm dd (irregular disc), white dorsal and ventral, any pattern bleached, hardly visible. DAP W:L 2:1, arm spine sequence 3, 3, 4, 4, 5, 5, 5, 5, 5, 4, 4, 4, 4, 4, 4, oral shield longer than wide, ventral disc completely granulated, $2^{\text {nd }}$ ventral arm spine longest, longer than an arm segment.

\section{Syntype ZMB Ech 962}

Collecting Data. Mozambique, $18^{\circ} 39.942 ’$ S 35 31.774’ E (from atlas), collector Peters, 1851.

Morphological Data. $13 \mathrm{~mm}$ dd, white dorsal and ventral disc, dorsal arms with brown bands, ventral arms white, DAP W:L 2:1, arm spine sequence 3, 3, 4, 5, 5, 5, 5, 5, 5, 5, 5, 5, 5, 5, 4, 4, 4, oral shield longer than wide, ventral disc completely granulated, $2^{\text {nd }}$ ventral arm spine longest, longer than an arm segment.

\section{Syntype ZMB Ech 963}

Collecting Data. Mozambique, $18^{\circ} 39.942^{\prime} \mathrm{S}$ 35³ 31.774’ E (from atlas), collector Peters, 1851.

MORPHOLOGICAL DATA. $13 \mathrm{~mm}$ dd, dorsally whitish-brown colour pattern, ventrally white, DAP W:L = 2:1, arm spine sequence $3,3,3,4,5,5,5,5,5,5,5,4,4,4,4,4$, 4, oral shield about as wide as long, ventral disc completely granulated, $2^{\text {nd }}$ ventral arm spine longest, longer than an arm segment.

\section{Syntypes ZMB 4660}

Collecting Data. Mozambique, Querimba Islands, $12^{\circ} 25.796^{\prime}$ S $40^{\circ} 35.948^{\prime}$ E (from atlas), collector Peters, 1851, 3 spms.

Morphological data. Spm A, 17-18 mm dd, white overall, DAP W:L 2:1, arm spine sequence 3, 3, 3, $4,4,5,5,5,5,5,5,5,4,4,4,4,4$, oral shield longer than wide; Spm B, $15 \mathrm{~mm}$ dd, white, DAP W:L 2:1, arm spine sequence $3,3,3,4,4,5,5,5,5,5,5,4,4,4,4,4$, 4, oral shield wider than long; Spm C,12 mm dd, white, DAP W:L 2:1, arm spine sequence 3, 3, 3, 4, 4, 5, 5, 5, 5, 5, 5, 5, 5, 5, 5, 5, 5, oral shield longer than wide; all specimens ventral disc completely granulated, $2^{\text {nd }}$ ventral arm spine longest, longer than an arm segment.

\section{Other material examined}

\section{SMNH-133230}

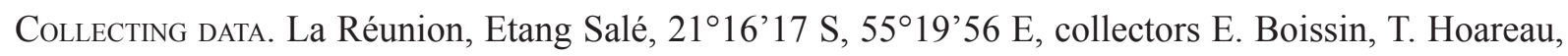
24 Feb. 2009. 1 spm.

MoRPHOLOGICAL DATA. $13 \mathrm{~mm}$ dd, dorsal disc grey-brown with a mosaic of irregular white spots (Fig. 2D), dorsal arms grey-brown with some dark spots, spines with dark longitudinal line, tips white, ventral disc white, grey-brown marbled, oral frame white, oral shields with dark brown spots, ventral arms white, arm spine sequence $3,3,3,4,4,5,5,5,5,5,4,4,4,4,4,4$, two dorsal spines shorter than arm width, 


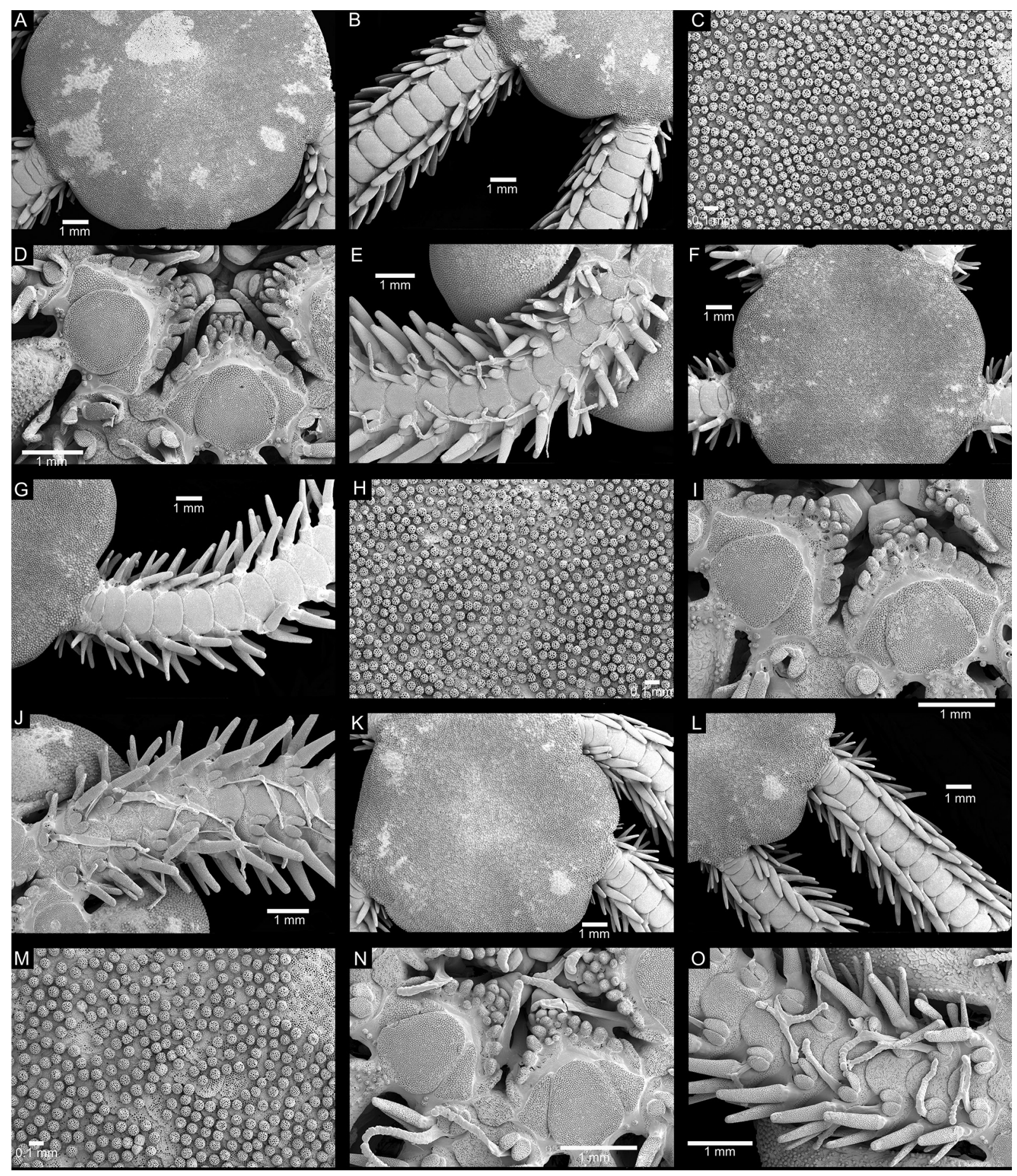

Fig. 4. SEM images of three species of Ophiocoma (Breviturma) subgen. nov. A-E. Ophiocoma brevipes Peters, 1851 (SMNH-133230). A. Dorsal disc. B. Dorsal arms. C. Dorsal disc granules. D. Jaws. E. Ventral arm. F-I. Ophiocoma dentata Müller \& Troschel, 1842 (SMNH-133232). F. Dorsal disc. G. Dorsal arm. H. Dorsal disc granules. I. Jaws. J. Ventral arm. K-N. Ophiocoma krohi sp. nov. paratype (SMNH-Type-8534). K. Dorsal disc. L. Dorsal arms. M. Dorsal disc granules. N. Jaws. O. Ventral arm. For institution codes see main text. 
$2^{\text {nd }}$ (and third) ventral spine longest. Ventral interradii fully granulated. Granule density on light image $248-280 \mathrm{~mm}^{-2}$.

Dorsal disc granule diameter $42-50 \mu \mathrm{m}$, granule density $240-248 \mathrm{~mm}^{-2}$, on SEM image (Fig. 4A, C); third spine about $1 \mathrm{~mm}$ long, proximal DAPs about $1.5 \mathrm{~mm}$ wide (Fig. 4B). Oral shield as wide as long; 6-8 tooth papillae, 5 lateral oral papillae at each jaw edge, outer papilla widest (Fig. 4D). Few granules on distal ends of oral shields. Two tentacle scales (Fig. 4E). Oral plates, adradial distal part with strong folds, abradial face with large muscle flange with horizontal striations, $2.4 \mathrm{~mm}$ wide, $2.0 \mathrm{~mm}$ high (Fig. 5A). Dental plate short, externally with two large tooth foramina in dorsal half, dorsalmost foramen oval, wider than long, other foramen almost round, internally each foramen divided by a wide septum, dorsalmost one into 2 long parallel slits, other one to 2 short oval holes; on external ventral half of dental plate two raised horizontal ridges, at edge a cluster of tiny depressions (Fig. 5D). Radial shield angular, internally with distal large round condyle (Fig. 5G). Adradial genital plate long, flat, thin, thickened at distal end, with condyle and pit; abradial genital plate thin, blade-like, curved distally (Fig. 5J). Proximal vertebrae $2.0 \mathrm{~mm}$ wide and $1.7 \mathrm{~mm}$ high (Fig. 5M).

\section{URUN 2013-11188}

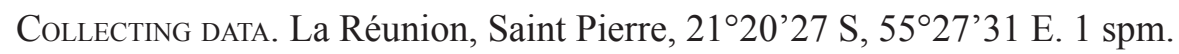

Morphological data. $19.7 \mathrm{~mm}$ dd. Arm spine sequence 3, 3, 3, 4, 4, 5, 5, 5, 5, 5, 5, 5, 5, 5, 5, 5, 5, 5, 4, 4, 4, 4, 4, 4. Granule density 176-188 $\mathrm{mm}^{-2}$.

\section{URUN 2008-04774}

Collecting DaTA. Madagascar, station MGNW-9, Sakatia Island, 1318’'16 S 4808’51 E. $1 \mathrm{spm}$.

Morphological Data. $13.08 \mathrm{~mm}$ dd. White with brown marbled pattern on dorsal and ventral disc, dorsal arms brown, oral frame and ventral arm white, few dark spots on oral and adoral shields. Granule density 148-168 $\mathrm{mm}^{-2}$. Arm spine sequence $3,3,3,4,4,5,5,5,5,5,4,4,4,4,4,4$.

\section{URUN 2008-05819}

Collecting Data. Madagascar, station MGNW-9, Sakatia Island, 13¹8’16 S, 4808’51 E. 1 spm.

MoRphological DATA. $13.5 \mathrm{~mm}$ dd. White-brown to grey marbled disc, grey arms with faint dark median line, oral frame and ventral arms white, spines mostly white. Granule density $180-200 \mathrm{~mm}^{-2}$. Arm spine sequence $3,3,3,4,4,5,5,5,5,5,5,4,4,4,4,4,4,4$.

\section{Remarks}

Ophiocoma brevipes is clearly distinguished from the other species of Breviturma subgen. nov. by its consistently white and light brown to grey colour pattern, up to five arm spines far out on the arms, and dorsal disc granule densities up to $250 \mathrm{~mm}^{-2}$. According to Devaney (1970), five spines occur in some specimens from $3 \mathrm{~mm}$ dd.

Ophiocoma (Breviturma) dentata Müller \& Troschel, 1842 Figs 2E, F, 4F-J, 5B, E, H, K, N, Q

Ophiocoma dentata Müller \& Troschel, 1842: 99, pl. 7, figs 3-3a.

Ophiocoma ternispina v. Martens, 1870: 252.

Ophiocoma insularia Lyman, 1862: 80.

Ophiocoma variegata Smith, 1876: 39.

non Ophiocoma marmorata - Devaney 1970: 17. 


\section{Diagnosis}

Species of Ophiocoma (Breviturma) subgen. nov. characterized by rarely possessing more than four arm spines and if present only at few segments; arm spines never annulated; variable in colour pattern (uniformly or marbled brown, reticulated of spotted); dorsal disc granule density less than $150 \mathrm{~mm}^{-2}$. Maximum size at least $28 \mathrm{~mm}$ dd (Devaney 1970).

\section{Type material examined}

\section{Holotype ZMB Ech 931}

Holotype of Ophiocoma dentata.

Collecting Data. Celebes (Sulawesi), $1^{\circ} 50.875^{\prime} \mathrm{S} 120^{\circ} 31.675^{\prime} \mathrm{E}$ (from atlas), collector Deppe.

MORPHOLOGICAL DATA. 18-19 mm dd (irregular disc), granules on dorsal disc mostly rubbed off, light and dark brown pattern on dorsal disc, dorsal arms medium brown, ventral interradii fully granulated, white, DAP W:L 2:1, arm spine sequence 3, 3, 4, 4, 4, 4, 4, 4, 4, 4, 4, 4, 3, 3, 3, 3, 3, oral shield longer than wide, ventralmost and $2^{\text {nd }}$ ventral spine longest, longer than an arm segment, dorsal spines thickened, ventral ones slender, not annulated.

\section{Holotype ZMB Ech 1815}

Holotype of Ophiocoma ternispina.

Collecting Data. Indonesia, Flores-Sea, Larantuka, Flores, $8^{\circ} 21.4^{\prime} \mathrm{N} 122^{\circ} 57.817^{\prime}$ E (from atlas), $1 \mathrm{spm}$.

Morphological Data. $16 \mathrm{~mm}$ dd, disc light brown, triangular brown spots and curved lines on DAPs, spines weakly annulated, DAP W:L 2:1, disc irregular round, ventral interradii completely granulated, arm spine sequence $3,3,3,(3) 4,4,4,(4) 3,3,3,3,3,3,3,3,3,3,3$ (numbers vary between arms), $2^{\text {nd }}$ ventral spine longest, slender spines, oral shield twice as long as wide.

\section{Syntype SMNH-Type-5244}

Syntype of Ophiocoma insularia.

Collecting Data. Hawaii, 1860, $1 \mathrm{spm}$ (originally donated to Stockholm by Museum of Comparative Zoology at Harvard).

Morphological Data. $17.6 \mathrm{~mm}$ dd, disc light brown marbled, arms light brown, dorsal and ventral side similar in colour, disc fully granulated dorsally and ventrally. No annulations on arm spines, spine sequence $3,3,3,4,4,4,5(4), 5(4), 5(4), 4,4,4,4,4,4,4,4,4,3,3,3$ (two arms with only 4 spines). Oral shield oval. Granule density $96-120 \mathrm{~mm}^{-2}$.

\section{Syntypes MCZN 1668}

Syntypes of Ophiocoma insularia.

Collecting Data. Hawaii, Maui, 1860, 7 spms.

Morphological Data. 13.3-16.6 mm dd, all dark brown to black on dorsal disc and arms, including spines, ventral side slightly lighter brown. Both middle spines about equal, longer than dorsal and ventral spines. (Photos provided by Adam Baldinger, MCZN).

\section{Syntypes MCZN 1666}

Syntypes of Ophiocoma insularia. 


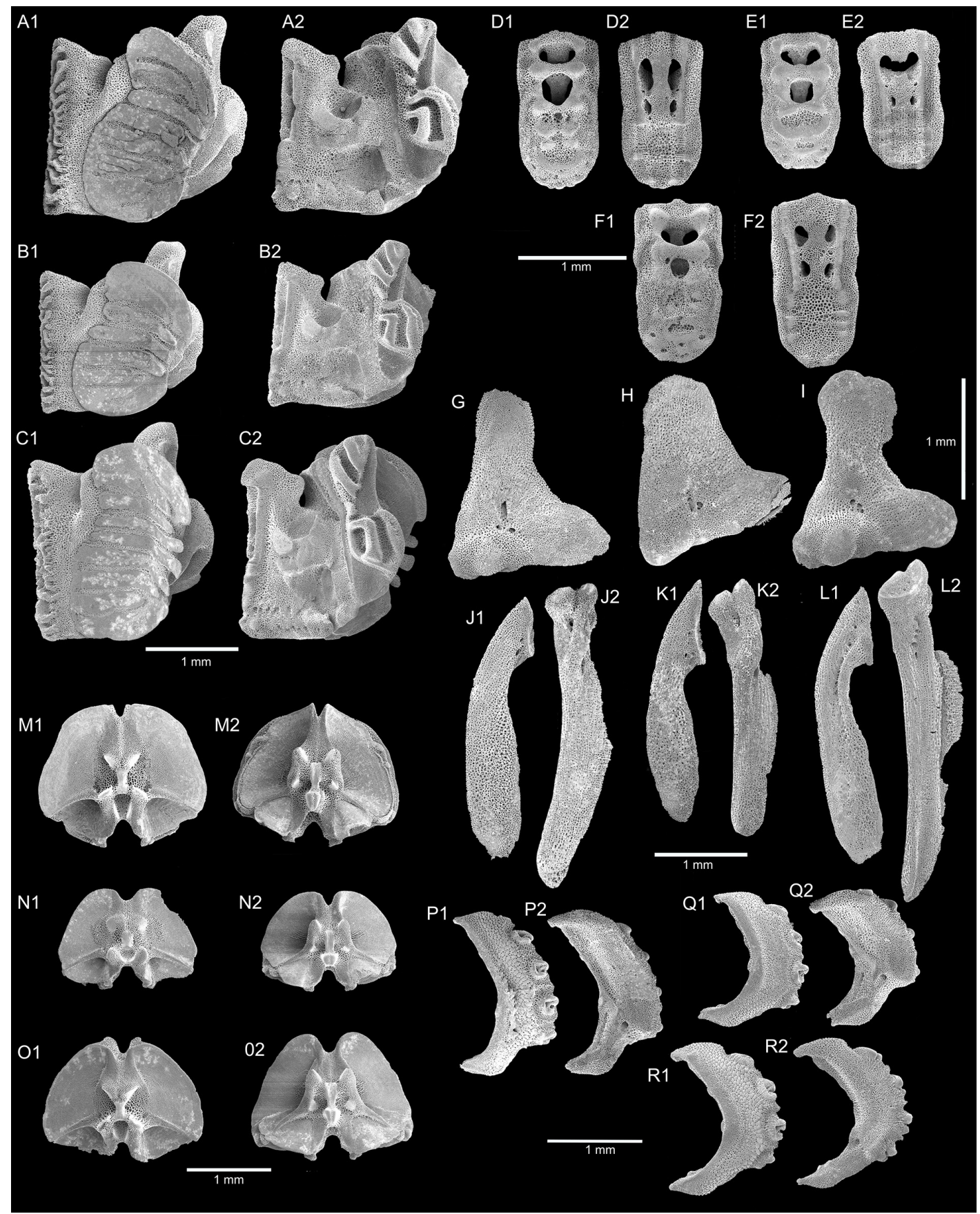

Fig. 5. SEM images of skeletal elements of Ophiocoma (Breviturma subgen. nov.) brevipes Peters, 1851 (SMNH-133230), O. (B.) dentata Müller \& Troschel, 1842 (SMNH-133232) and O. krohi sp. nov. paratype (SMNH-Type-8535). A-C. Oral plates, abradial aspect at left, orientated with dorsal side up. A. O. brevipes. B. O. dentata. C. O. krohi sp. nov. D-F. Dental plates, external aspect left, internal aspect right, dorsal edge upwards. D. O. brevipes. E. O. dentata. F. O. krohi sp. nov. G-I. Radial shields, internal aspect, proximal edge upwards. G. O. brevipes. H. O. dentata. I. O. krohi sp. nov. J-L.Vertebrae from proximal part of arm, proximal aspect left, dorsal upwards. J. O. brevipes. K. O. dentata. L. O. krohi sp. nov. M-O. Genital plates, abradial plate left, adradial plate right, distal ends up (towards radial shields). M. O. brevipes. N. O. dentata. O. O. krohi sp. nov. P-R. Lateral arm plates from proximal arm, external aspect left, internal aspect right, distal end right. P. O. brevipes. Q. O. dentata. R. O. krohi sp. nov. For institution codes see main text. 
Collecting Data. Hawaii, 1860, 2 spms.

MoRPhOlOGical Data. 19.5 and $31.6 \mathrm{~mm}$ dd, dark brown disc and arms, with lighter abraded patches on dorsal disc, dark brown on ventral disc and arms; a note among the labels reads "4-5 spines, 3 upper about equal". (Photos provided by Adam Baldinger, MCZN).

\section{Other material examined}

SMNH-133232

Collecting Data. La Réunion, Etang Salé, 2116’17” S 55¹9’56” E, collectors E. Boissin, T. Hoareau, 24 Feb. 2009, 1 spm.

MORPHOLOGICAL DATA. $11 \mathrm{~mm}$ dd, dorsal disc with a brown multipointed star-shape, white centre spot with brown dots, interradii white with brown marbled pattern (Fig. 2E); dorsal arm white with rectangular dark brown outlines, spines brown at base, fading into white towards their tip. Ventral interradii brown and white marbled, fully granulated to oral shields, oral frame white, oral shields with 2 parallel longitudinal brown lines. Ventral arm white with median brown dots and short lines. Arm spine sequence 3, 3, 3, 3, $4,4,4,4,4,4,3,3,3,3,3,3$. Granule density $216-224 \mathrm{~mm}^{-2}$.

Dorsal disc granule diameter 49-54 $\mu \mathrm{m}$, granule density 120-144 $\mathrm{mm}^{-2}$, on SEM image (Fig. 4F, H); third spine on proximal segments $1 \mathrm{~mm}$ long, DAPs 1.4-1.6 mm wide Fig. 4G). Oral shields as wide as long; 5-6 tooth papillae, 5 lateral oral papillae at each jaw edge, outer papilla widest (Fig. 4I). Few granules on proximal ends of oral plates. Oral plates, adradial distal part with strong folds, abradial face with large muscle flange with horizontal striations (Fig. 5B), $1.6 \mathrm{~mm}$ wide, $2.0 \mathrm{~mm}$ high. Dental plate short, externally with two large tooth foramina in dorsal half, dorsalmost foramen oval, wider than long, other foramen square; internally each foramen divided by a wide septum, dorsalmost one into two wide vertical oval slits (septum broken), other foramen into two short parallel holes (Fig. 5E). External ventral half of dental plate with smooth horizontal ridges, forming two wide ovals, the ventralmost one half the size of the other; a cluster of tiny depressions at ventral end of plate (Fig. 5E1). Radial shield triangular with concave proximal edge, internally with distal round condyle (Fig. 5H), not as ball-like pronounced as in $O$. brevipes and $O$. krohi sp. nov. Adradial genital plate (Fig. 5K1) long, flat, thin, thickened at distal end, with condyle and pit; abradial genital plate (Fig. 5K2) thin, blade-like, curved distally. Proximal vertebrae $1.6 \mathrm{~mm}$ wide and $1.2 \mathrm{~mm}$ high (Fig. 5N).

\section{URUN 2009-11047}

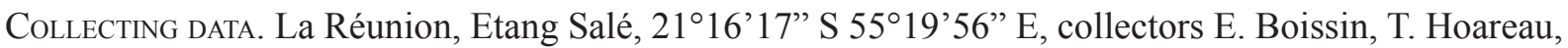
24 Feb. 2009, 1 spm.

Morphological DATA. $23.7 \mathrm{~mm}$ dd, dorsal disc uniformly reddish brown (Fig. 2F), dorsal arm dark brown, ventral disc and arms dark brown. Granule density 44-60 $\mathrm{mm}^{-2}$, granule diameter 70-90 $\mu \mathrm{m}$. Arm spine sequence $3,3,3,4,4,4,4(5), 4(5), 4,4,4,4,4,3,3,3,3$ (5 spines on one side of one arm, once at segment 7 and once at segment 8$)$.

\section{URUN 2007-00380}

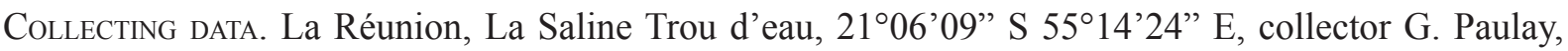
$2007,1 \mathrm{spm}$.

MoRPHOLOGICAL DATA. $24.2 \mathrm{~mm}$ dd, dorsal disc brown, dorsal arms dark brown with widely spaced light markings, spine slighter brown, ventral disc brown, oral frame lighter, ventral arms brown. Granule density 52-68 $\mathrm{mm}^{-2}$. Arm spine sequence 3, 3, 3, 4, 4, 4, 4, 4, 4, 4, 4, 4, 3, 3, 3, 3, 3 . 


\section{Remarks}

The types of $O$. insularia are clearly all conspecific. Their colouration and large size suggest that they are also conspecific with $O$. dentata. Ophiocoma dentata occurs in several colour morphs, from uniformly dark brown to brown marbled discs with banded arms, and reticulated or spotted forms that resemble $O$. doederleini. It can always be distinguished from the latter species by the absence of annulations on the arm spines, and shorter arm spines. In addition, only the largest individuals sometimes have more than four spines on few arm segments. The conspecificity of the various colour morphs is also confirmed by molecular data (Boissin \& Hoareau, unpublished). Another species currently believed to be conspecific with $O$. dentata is $O$. variegata with type locality Rodriguez Island. According to the original description (Smith 1876), the type measured $28 \mathrm{~mm}$ dd and had only four arm spines, which agrees with $O$. dentata.

Devaney (1970) synonymized $O$. marmorata with $O$. dentata, but as we discuss below, the holotype of $O$. marmorata is most likely not conspecific with $O$. dentata.

Ophiocoma (Breviturma) krohi sp. nov.

urn:1sid:zoobank.org:act:D662D2A3-330D-4693-8757-E6112C4BABC1

Figs 2G-L, 4K-O, 5C, F, I, L, O, R, 6

Ophiocoma brevipes - Stöhr et al. 2008: 553.

Ophiocoma sp. nov. - Hoareau et al. 2013: 2.

\section{Diagnosis}

Species of Ophiocoma (Breviturma) subgen. nov. with variegated to uniform dark brown colour pattern; arm spines may be weakly annulated, but not as clearly light and dark banded as in $O$. doederleini; up to four arm spines (in large specimens beyond segment 15), sometimes five at segments 6-9 (rarely 5-8); relatively coarse disc granulation. Maximum size about $16 \mathrm{~mm}$ dd.

\section{Etymology}

The species is named in honour of Dr. Andreas Kroh, Vienna, for his important contributions to echinoderm systematics.

\section{Type material}

\section{Holotype}

La Réunion Island, St. Pierre, 21²0’27 S 55²7’31 E, collectors E. Boissin, T. Hoareau, 25 Mar. 2009, in ethanol (MNHN-IE-4300).

\section{Paratypes}

From same locality as holotype 8 spms: $1 \mathrm{spm}$, dissected, fragments on SEM stubs and arms in ethanol (SMNH-Type-8535); 1 spm gold coated and uncoated arms dry (SMNH-Type-8534); 2 spms in ethanol (SMNH-Type-8531, 8536); $1 \mathrm{spm}$ in ethanol (UF13938); $1 \mathrm{spm}$ in ethanol (URUN 2009-11108); $2 \mathrm{spms}$

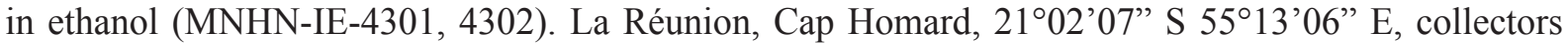
E. Boissin, T. Hoareau, 23 Feb. 2009: 2 spms in ethanol (SMNH-Type-8532, 8533); 1 spm in ethanol (MNHN-IE-4303).

\section{Description}

\section{Holotype}

$12.7 \mathrm{~mm}$ dd, radial shields completely obscured by granules, granule density $104 \mathrm{~mm}^{-2}$. Arm spine sequence $3(2), 3(2), 3,4,4,4,4,4,4,4,4,3,3,3,3$. Dorsal disc with dark and lighter brown lines 
radiating from a dark central patch, some white spots at disc edge (Fig. 6A). Dorsal arms medium brown with dark and white transverse bands (Fig. 6B). Ventral disc dark brown mottled, ventral arms and oral frame lighter brown, oral shields dark. Ventral interradii completely, but sparsely granulated (Fig. 6D, E). Dorsal spines brown, ventral spines weakly annulated brown on white. DAPs oval, W:L 1.8:1, contiguous. Dorsalmost spine a little longer than a segment, middle spines about 2 segments long, ventralmost spine slightly shorter. Arm spines flattened, slightly tapered with truncated tips, dorsalmost spine wider than other spines. Oral shields trapezoid, distally almost as wide as long, proximally just over half as wide as long, lateral edges straight (except madreporite), proximal and distal edges convex (Fig. 6D). Madreporite larger than other oral shields, more rounded, all edges convex. At tip of jaw a cluster of 8-10 small, round tooth papillae. Along each jaw edge 4 rectangular, wider than high, oral papillae, and distally a smaller papilla at an angle to the others (Fig. 6E). Adoral shields uneven triangular, framing the lateral edges of the oral shield, not meeting proximally. Bursal slits extend from oral shield to disc edge. First ventral arm plate outside mouth-slit, small, winglike pentagonal, middle of distal edge notched. Following plates pentagonal, about as long as greatest width, with obtuse proximal angle, lateral edges notched, distal edge convex, contiguous (Fig. 6C). 2 oval scales at a slightly open angle at the lateral arm plate of each tentacle pore proximally; the inner scale decreases gradually and is absent on the distalmost segments. Tube feet expanded, smooth, with distal constrictions and terminal bulb.

The paratypes are similar to the holotype in most characters, except as follows:

\section{SMNH-Type-8534}

$1 \mathrm{spm}, 11 \mathrm{~mm}$ dd, dark brown radiating stripes on dorsal disc, interradii mottled light and dark brown, overall small white dots; dorsal arms dark brown with some lighter markings, spines white with diffuse brown pattern, some with annulations (not figured). Ventral interradii dark brown mottled with white, fully granulated; adoral shields and oral plates unevenly brown, oral shields dark brown with white edges, oral papillae white. Ventral arm plates and tentacle scales brown with white edges. Arm spine sequence 3, 3, 3, 4, 4, 5, 5, 4, 4, 4, 4, 4, 4, 4, 4, 4. Granule density 132-136 $\mathrm{mm}^{-2}$ on light image.

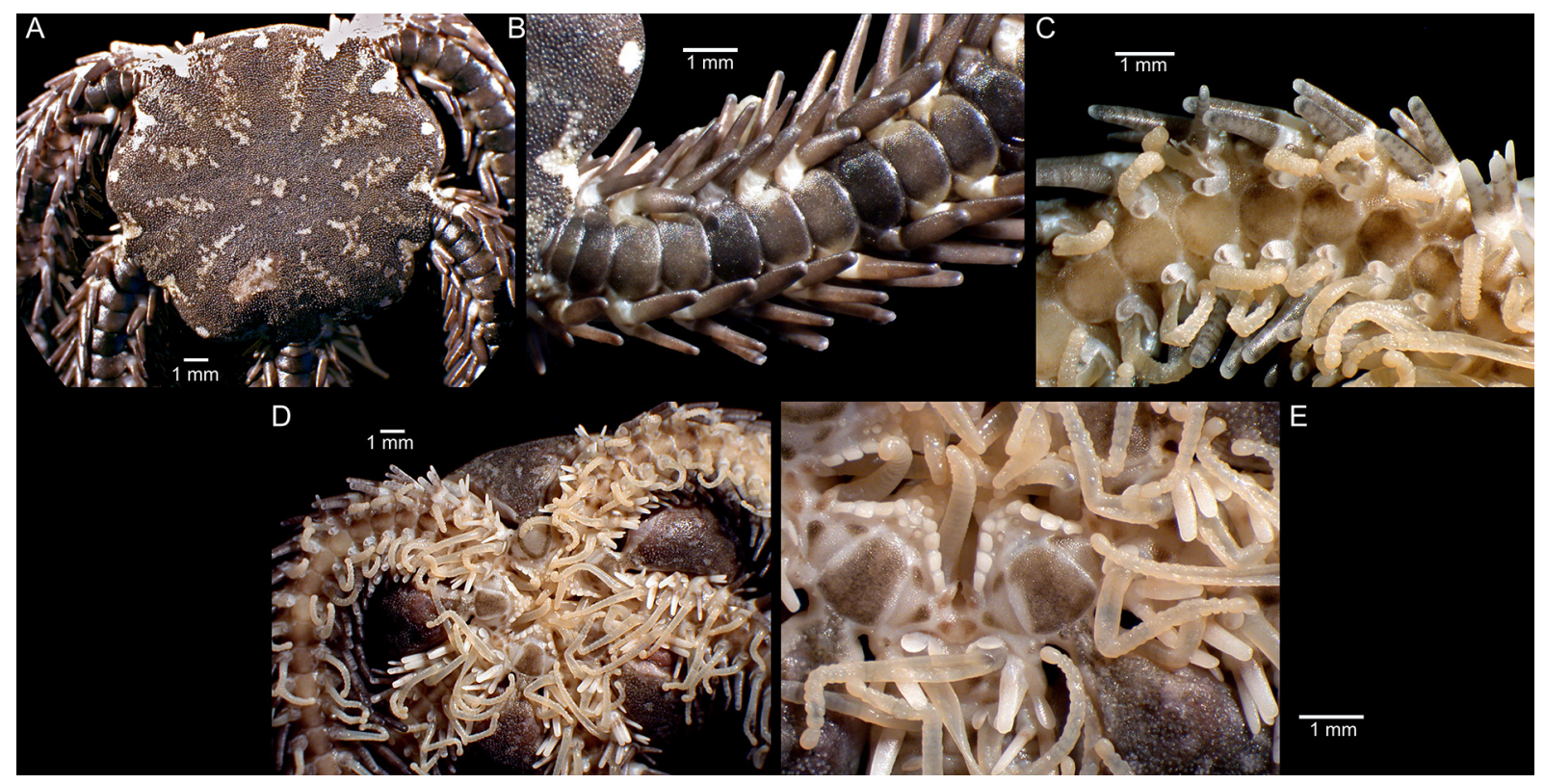

Fig. 6. Holotype of Ophiocoma (Breviturma subgen. nov.) krohi sp. nov. (MNHN-IE-4300). A. Dorsal disc. B. Dorsal arm. C. Ventral arm, note the pattern on the arm spines. D. Ventral disc. E. Jaws. 
Dorsal disc granule diameter 57-73 $\mu \mathrm{m}$, granule density $132-136 \mathrm{~mm}^{-2}$, on SEM image (Fig. 4K, M); third arm spine $1.3 \mathrm{~mm}$ long, DAP $1.2 \mathrm{~mm}$ wide (Fig. 4L). Oral shields as wide as long (Fig. 4N). 5 tooth papillae, 5 lateral oral papillae at each jaw edge, outer papilla widest. Few granules on proximal ends of oral plates (Fig. 4N).

\section{SMNH-Type-8535}

$1 \mathrm{spm}, 13 \mathrm{~mm}$ dd, dorsal disc dark brown, white spots on radial shields; dorsal arms banded dark and light brown; spines dark brown, on distal arm annulated (not figured). Granule diameter 57-74 $\mu \mathrm{m}$, density $104 \mathrm{~mm}^{-2}$. Ventral interradii dark brown; adoral shields light brown with dark spots; oral shields dark brown with white edges; oral papillae white. Ventral arm plates brown with white edges and lighter areas. Arm spine sequence 3, 3, 3, 4, 4, 5, 5, 4, 4, 4, 4, 4, 4, 4, 4, 4, 4. Up to 5 tooth papillae, 5 lateral oral papillae at each jaw edge, outer papilla widest. Few granules on distal ends of oral plates.

Oral plates, adradial distal part with strong folds, abradial face with large muscle flange with horizontal striations that end in deep incisions in the distal edge; $1.9 \mathrm{~mm}$ wide, $2.3 \mathrm{~mm}$ high (Fig. 5C). Dental plate short, with two tooth foramina on external dorsal half, dorsalmost one oval, wider than long, other small, round; internally a wide septum divides each foramen into two pairs of vertical oval holes, upper ones larger than lower ones (Fig. 5F). Radial shield angular, with large round condyle at internal distal end (Fig. 5I). Adradial genital plate long, flat, thin, thickened at distal end, with deep pit; abradial genital plate thin, blade-like, curved distally (Fig 5L). Proximal vertebrae $2.0 \mathrm{~mm}$ wide and $1.5 \mathrm{~mm}$ high (Fig. 5Q).

\section{SMNH-Type-8531}

$1 \mathrm{spm}, 6.8 \mathrm{~mm}$ dd, granule density $152 \mathrm{~mm}^{-2}$. Arm spine sequence 3, 3, 3, 4, 4, 5, 5, 4, 4, 4, 4, 3, 3, $3,3,3,3$. Dorsal disc with central dark brown patch, with white central spot, dark brown radiating lines, medium brown interradial lines on light brown background, white spots at disc edge (Fig. 2I). Dorsal arms with transverse dark and lighter brown bands. Ventral disc light brown with irregular cream patches, oral frame and ventral arms lighter brown, oral shields dark.

\section{SMNH-Type-8532}

$1 \mathrm{spm}, 9.6 \mathrm{~mm}$ dd, granule density 116-120 $\mathrm{mm}^{-2}$. Arm spine sequence 3, 3, 3, 4, 4, 4, 4, 4, 4, 4, 4, 4, 3 , $3,3,3$. Dorsal disc dark brown mottled, dorsal arms lighter brown with dark bands. Ventral disc light and dark brown mottled (not figured). Ventral arms and oral frame light brown, oral shields with dark patch.

\section{MNHN-IE-4303}

$1 \mathrm{spm}, 8.1 \mathrm{~mm}$ dd, granule density $124 \mathrm{~mm}^{-2}$. Arm spine sequence 3, 3, 3, 4, 4, 4, 4, 4, 4, 4, 4, 4, 4, 3, $3,3,3$. Dorsal disc light brown mottled, dorsal arms light brown with dark bands. Ventral disc light and dark brown mottled, arms and oral frame crème, oral shields with dark patches.

\section{SMNH-Type-8533}

$1 \mathrm{spm}, 10 \mathrm{~mm}$ dd, granule density 140-164 $\mathrm{mm}^{-2}$. Arm spine sequence 3, 3, 3, 4, 4, 5, 5, 5, 5, 4, 4, 4, 4, $4,4,4,4,4,4,4,4,3,3,3$. Dorsal disc variegated white and brown, arms dark and lighter brown banded, spines annulated (Fig. 2G).

\section{SMNH-Type-8536}

$1 \mathrm{spm}, 15.5 \mathrm{~mm}$ dd, granule density 64-96 $\mathrm{mm}^{-2}$. Arm spine sequence 3, 3, 3, 4, 4, 4, 4, 4, 4, 4, 4, 4, 4, 4, 4, 4, 4, 4, 4, 4, 3, 3, 3. Dorsal disc uniformly dark brown, except on radial shields white granules (Fig. 2K). DAPs dark brown, all spines weakly annulated with irregular dark stripes on white. Oral frame white with dark marks on oral shields. Ventral arm plates dark brown with white borders. 
MNHN-IE-4302

$1 \mathrm{spm}, 13 \mathrm{~mm}$ dd, granule density 96-104 $\mathrm{mm}^{-2}$. Arm spine sequence 3, 3, 3, 4, 4, 4, 4, 4, 4, 3, 3, 3, 3, 3,3 . Light brown disc with irregular dark spots or blotches. Dorsal arms medium brown with widely spaced dark brown transverse bands (Fig. 2H). Ventral disc medium and dark brown mottled, ventral arms and oral frame light brown to cream, oral shields with dark pattern.

\section{MNHN-IE-4301}

$1 \mathrm{spm}, 11.8 \mathrm{~mm}$ dd, granule density $72-96 \mathrm{~mm}^{-2}$. Arm spine sequence 3, 3, 3, 4, 5, 5, 5, 5(4), 4, 4(5), 4, 4, 4, 4, 4, 4, 4, 4, 4, 3, 3, 3. Dorsal disc dark brown mottled, dorsal arms dark brown with few white marks, spines dark brown (Fig. 2J). Oral frame cream white with light brown marks on oral shields, ventral arm plates brown.

\section{URUN 2009-11108}

$1 \mathrm{spm}, 11.3 \mathrm{~mm}$ dd, granule density $112-120 \mathrm{~mm}^{-2}$. Arm spine sequence 3, 3, 3, 4, 4, 4, 4, 4, 4, 4, 4, 4, $4,3,3,3,3,3,3,3,3$. Dorsal disc with dark brown star-shaped pattern, interradii brown with irregular white spots, irregular white spots in centre of disc, white granules on radial shields (not figured). Dorsal arms mostly dark brown, some lighter patches, spines dark brown. Oral frame white, brown patches on oral shields. Ventral arm plates brown with white margins.

\section{UF13938}

$1 \mathrm{spm}, 10.3 \mathrm{~mm}$ dd, granule density $112-116 \mathrm{~mm}^{-2}$. Arm spine sequence $3,3,3,4,4,5,5,5,5(4), 4,4,4$, $4,4,3,3,3$. Dorsal disc light brown with irregular dark patches and spots, dorsal arm light brown with widely spaced narrow dark brown bands (Fig. 2L). Spines light brown, weakly annulated. Oral frame white with light brown pattern on oral shields, ventral arm plates light brown with white margins.

\section{Remarks}

Ophiocoma krohi sp. nov. differs from $O$. brevipes and $O$. dentata in maximum size, granule size and density, and in colour pattern. Although quite variable in colour, it has never been observed with reticulated or spotted pattern as $O$. dentata and O.doederleini. We examined 12 specimens and half of them showed up to five arm spines on 2-4 segments, the other half had only four. Thus they all differ in spine number from $O$. brevipes, but some are similar to $O$. dentata in lacking a fifth spine, although they may have four spines farther out on the arm than $O$. dentata.

The specimens reported by Stöhr et al. (2008) are most likely conspecific with $O$. krohi sp. nov. based on colour pattern, the presence of five spines and their small size. The species has a wide geographic distribution on coral reefs across the Indian and Pacific Oceans at depths of 0-30 m (Hoareau et al. 2013).

non-Breviturma, unclear subgenus

Ophiocoma marmorata Marktanner-Turneretscher, 1887

Ophiocoma marmorata Marktanner-Turneretscher, 1887: 303, pl. 12 figs 16, 17. non Ophiocoma dentata - Devaney 1970: 17.

\section{Material examined}

NHMW 3. Zool. Abt. Nr. 10.466

Collection Eichhorn $2873,0-7^{\circ} \mathrm{N}, 23-25^{\circ} \mathrm{W}$ (= Tropical Atlantic). Lot of 5 spms, wrongly registered as "syntypes". One very obviously belongs to another species and was not examined more closely. Examined, photographed and data transmitted by A. Kroh, Vienna. Each spm is treated separately below. 


\section{Description}

\section{Holotype NHMW 10.466a}

$8.7 \mathrm{~mm} \mathrm{dd}$, dorsally light orange colour pattern with darker spots on arms, ventrally cream coloured, spines white, not annulated, arm spine sequence (2)3, 3, 3, 3(4), 4, 4, 4, 3(4), 4, 3(4), 4(3), 3(4), 4(3), $3,4,3$ (numbers vary between arms and alternate between segments), oral shield longer than wide. Granule density about $80 \mathrm{~mm}^{-2}$. Holotype status inferred from original description.

\section{NHMW 10.466b}

$6.3 \mathrm{~mm} \mathrm{dd}$, cream colour dorsal and ventral, no pattern, coarser granules than holotype, arm spine sequence $3,3,3(4), 4,4,4,4,4,4,4,4,4,4,4,4$. Granule density $60 \mathrm{~mm}^{-2}$.

\section{NHMW 10466c}

$6.1 \mathrm{~mm} \mathrm{dd}$, cream colour dorsal and ventral, no pattern, coarser granules than holotype, arm spine sequence $3,3,4,4,4,4,4,4,4,4,4,4,4,4,4$. Granule density $48 \mathrm{~mm}^{-2}$.

\section{NHMW 10.466d}

$5.2 \mathrm{~mm} \mathrm{dd}$, cream colour dorsal and ventral, no pattern, coarser granules than holotype, arm spine sequence 3, 3, 4, 4, 4, 4(5), 4(5), 4, 4, 4, 4, 4, 4, 4, 4. Granule density $60 \mathrm{~mm}^{-2}$.

\section{Remarks}

The original description of $O$. marmorata mentions only a single specimen of $9 \mathrm{~mm}$ dd (MarktannerTurneretscher 1887), which should be regarded as the holotype. The remaining three specimens cannot be regarded as type material since they are not mentioned in the description. The locality of $O$. marmorata is most likely incorrect as it lies in the middle of the Atlantic Ocean at great depth. Ophiocoma is a shallow water tropical genus, rarely reported below $100 \mathrm{~m}$ depth. Taking into account that the determination of coordinates, in particular longitude, was difficult and often inaccurate in the $19^{\text {th }}$ century, we think it possible that the correct locality may be the Saint Peter and Saint Paul Archipelago, which is situated at $0.9^{\circ} \mathrm{N}, 29^{\circ} \mathrm{W}$, assuming that the data are based on the Greenwich Meridian. Instead, the Ferro Meridian may have been used, which would put the locality at $40^{\circ} 40^{\prime}-42^{\circ} 40^{\prime} \mathrm{W}$ today, even closer to Brazil. The specimens were bought by director Eichhorn of Graz (Austria) from the trader Hugo Schilling in Hamburg in 1882, together with several other echinoderms, and transferred to the natural history museum in Vienna (A. Kroh, pers. comm.).

The alternating spine sequence of the holotype of $O$. marmorata may be interpreted as an unusual variation, if we accept that it is conspecific with the three smaller specimens in the lot. This character is typical for species in the scolopendrina group though and therefore the holotype of $O$. marmorata may belong to one of the species in that group, possibly O. echinata (Lamarck, 1816) or O. wendtii Müller \& Troschel, 1842, which are the only two species of that group known from the Atlantic Ocean. The smaller specimens, however, may belong to a different species, possibly $O$. pumila Lütken, 1856, a member of the pumila group, which has a spine sequence similar to the smaller O. marmorata (NHMW 10.466b-d) and is known from the Atlantic. Devaney (1970) does not seem to have examined O. marmorata and did not know about the existence of five spines on one of the specimens in the lot. He also did not consider the significance of geographic distribution when he suggested that $O$. marmorata (an Atlantic species) may be conspecific with $O$. dentata (an Indo-Pacific species). Although the fifth arm spines in the small O. marmorata are in exactly the same position, arm segments 6 and 7, as in $O$. krohi sp. nov., the granules in these small specimens are high and pointed, different from those found in the species of Breviturma subgen. nov. Granules like that are known from the pumila group. The holotype of O. marmorata has low round granules, which also supports that it is a different species from the three smaller specimens. 
The taxonomic status of $O$. marmorata could not be completely resolved by this study and requires further investigation, but we strongly doubt that it is conspecific with $O$. dentata. We propose that the synonymy of $O$. dentata includes only $O$. ternispina, $O$. insularia and $O$. variegata.

\section{Remarks on species delimitations}

Prior to Devaney's (1970) work, the three species previously recognized in the brevipes group were considered mere variations of a single species. Devaney (1970) was able to separate them by spine sequence, colour pattern and arm width. In addition to the annulated colour pattern of the arm spines, spine numbers seem to be a critical character to distinguish between $O$. dentata and $O$. doederleini. According to Devaney (1970), O. dentata has five arm spines only at disc diameters above $20 \mathrm{~mm}$ and spine numbers decrease to three on the middle arm (beyond segment 17 at 13-14 $\mathrm{mm}$ dd, beyond segment 25 at $24 \mathrm{~mm} \mathrm{dd}$ ), whereas four spines are found on far more distal arm segments in $O$. doederleini of similar disc sizes (to segment 32-38 at 13-14 mm dd, to segment 55 at $24 \mathrm{~mm} \mathrm{dd}$ ). In light of our findings (Table 1) we cannot exclude though that the specimens regarded as O. dentata by Devaney actually included individuals of $O$. krohi sp. nov.. All of his data must therefore be treated with caution. The type of $O$. dentata (18-19 $\mathrm{mm}$ dd) has up to four spines on the proximal arm segments, decreasing to three from segment 13, which contradicts Devaney's (1970) observations. Our largest specimen of O. dentata has two occurrences of five spines among all ten sides of the arms, and three spines from segment 16.

In the type material of $O$. brevipes, spine numbers appear to be uncorrelated with disc size; five spines are present from the fourth, fifth or sixth segment and for a variable number of segments before the number drops to four and finally three again on the distalmost part of the arm. The smallest type, at $12 \mathrm{~mm} \mathrm{dd}$, shows 12 segments with five spines, whereas the largest, at 17-18 mm dd shows only six segments with five spines; in both animals there are five spines from the sixth segment. Similarly, spine numbers vary between specimens on the types of the three nominal species currently regarded as conspecific with

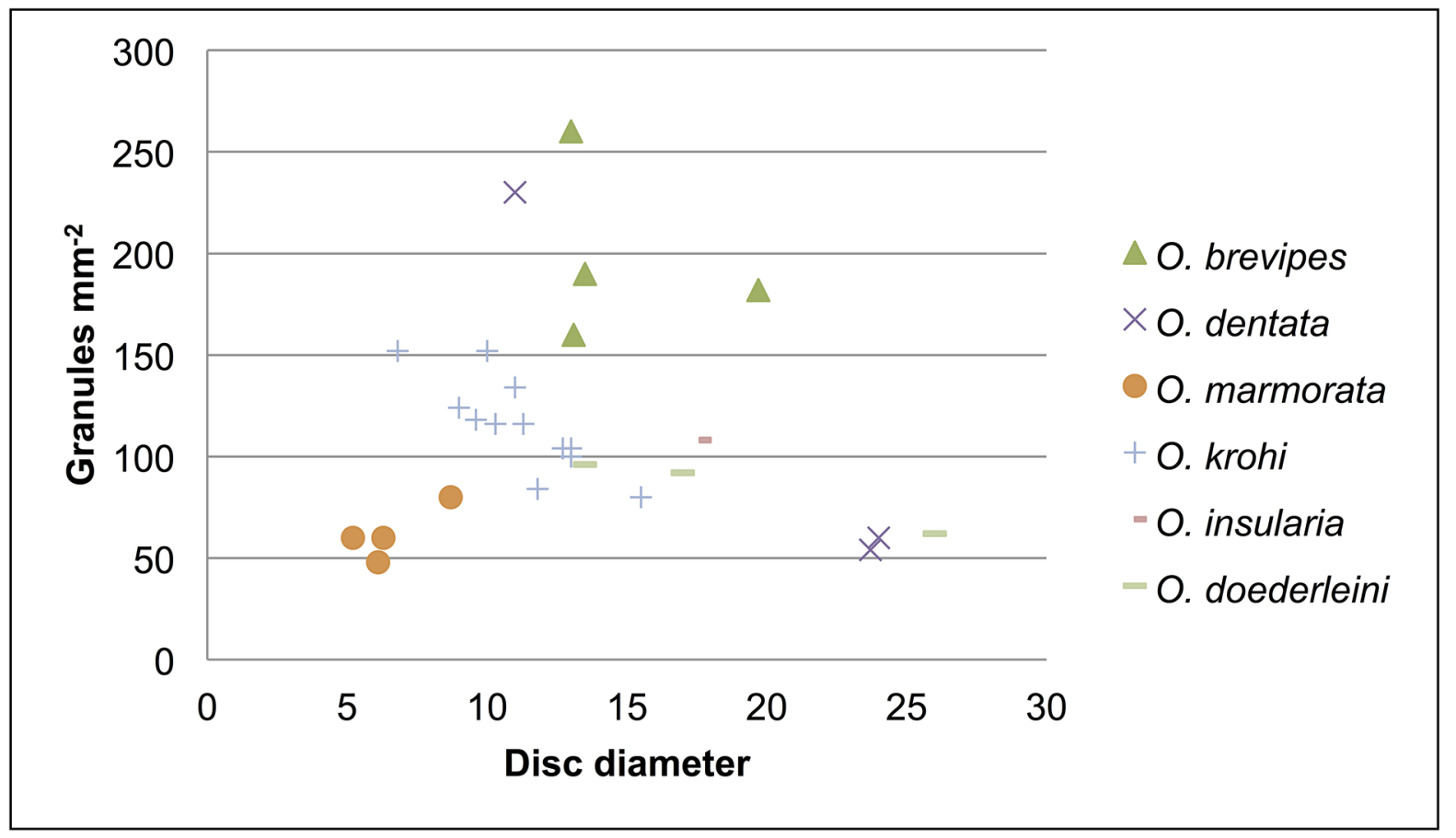

Fig. 7. Granule densities in relation to disc diameter of the examined nominal species of Ophiocoma, from Table 1. 
O. dentata. In $O$. krohi sp. nov., the smallest specimen has five spines on two segments, while the largest one has only up to four spines. These variations are common in the genus Ophiocoma as was observed already by Devaney (1970). We also found significant differences in granule density among species as shown in Fig. 7 (Kruskal-Wallis test, $\mathrm{K}=8.9055$, d.f. $=3, \mathrm{P}=0.0306$ ). Likewise, granule sizes and/or densities may be subject to growth changes as suggested by the inverse correlation observed between the granule density and the disc diameter in $O$. krohi sp. nov. (Spearman test, $R=-0.8012, P=0.0006$ ). The smallest $O$. krohi sp. nov. had 152 granules $/ \mathrm{mm}^{2}$, whereas the largest one had only 64-96 granules/ $\mathrm{mm}^{2}$. Small $O$. dentata have three times higher granule densities as large ones, which suggests that granule numbers do not increase with growth. Somewhat contradictory are the data for $O$. brevipes, where specimens from La Réunion have greater granule densities than similar size specimens from Madagascar.

We decided to examine a rarely used character, granule density, that has occasionally been mentioned in species descriptions and revisions, but never been used in a systematic way to differentiate Ophiocoma species. Devaney (1970) used granule densities as a character in his key to the species of the scolopendrina group, but only for few of the species. Granule densities were compared between light images and SEM images of the same animal and the small observed differences can probably be attributed to uneven distribution of the granules on the disc, rather than to a real difference in accuracy between these methods. Our method of counting granules on digital images resulted in considerably higher numbers than what has been published before. However, published values are not always related to size and as we see in our results, the largest specimens have larger and fewer granules. It is also possible that counts on images are more accurate than counts under the dissecting microscope. In any case, since we used the same method for all specimens, the differences between them are valid and important.

Devaney (1970) examined arm span on the $10^{\text {th }}$ free segment, "composed of the length of the longest arm spines on each side of a segment, the breadth of the dorsal arm plate and the breadth of the lateral arm plates" (cited from Devaney 1970), and found larger arm spans in O. doederleini than in O. dentata. However, his values overlap and vary uncorrelated to size, and we consider this a weak character that is difficult to assess.

\section{Discussion}

\section{Phylogeny}

The molecular phylogeny suggests monophyly of the brevipes group with respect to the scolopendrina group, which supports the establishment of Breviturma subgen. nov. This is the first time that the molecular basis of the species groups of Ophiocoma described by Devaney (1970) can be demonstrated. The phylogeny also revealed four deep lineages within Breviturma subgen. nov. corresponding to the three known species and the new species described here. Within this subgenus, $O$. doederleini is the most distantly related, and the new species $O$. krohi seems to be sister taxon to the $O$. brevipes/O. dentata clade, even though the data are not strongly supported.

\section{Morphology}

Devaney (1970) showed that the dental plate can be used to divide the genus Ophiocoma into groups. All our specimens concur with his description of the dental plate in the brevipes group, about twice as long as wide with small part for tooth granules, and all tooth foramina divided by a wide septum. The relationships within Breviturma subgen. nov. are difficult to resolve with morphological means. Benavides-Serrato \& O'Hara (2008) showed that the dental plate in the scolopendrina group differs between species, but that does not seem to hold true within Breviturma subgen. nov. There appear to be slight differences in the geometry of the oral plates, but it is currently unclear if these are significant. The overall shape of the oral plate is the same in all species, but it seems to be lower and shorter in $O$. dentata 
than in $O$. brevipes and $O$. krohi sp. nov. Similarly, the geometry of the vertebrae seems to differ slightly, with $O$. dentata having the smallest vertebra. However, these were not taken from exactly the same arm segment and size differences may correlate with ontogenetic age and overall size of the animal.

As with the molecular data, Ophiocoma doederleini is clearly distinguished from the other three species by morphology. The most conspicuous difference is the presence of annulated arm spines. The spines are also longer and thinner than in the other species. Granule density of the smallest specimen concurs with both $O$. dentata and $O$. brevipes, while the larger specimens have densities similar to $O$. dentata. It is most similar in arm spine numbers to $O$. krohi sp. nov. which also exhibits weakly annulated spines. However, the new species reaches a much smaller size $(16 \mathrm{~mm}$ dd vs. $26 \mathrm{~mm}$ dd) and differs in disc colour patterns (Fig. 2G-L).

Ophiocoma brevipes and $O$. krohi sp. nov. are similar in having up to five arm spines at small sizes, but differ in the number of segments that have five spines; they are similar also in the shape of the radial shield with a large ball-like articulation condyle that is less pronounced in $O$. dentata and $O$. doederleini. The genital plates of the three most closely related species $O$. brevipes, $O$. dentata and $O$. krohi sp. nov. are similar, but in $O$. dentata the adradial genital plate is relatively shorter and the abradial genital plate has a wider proximal edge, similar to $O$. doederleini. In granule density, $O$. dentata is close to $O$. brevipes, but both differ greatly from $O$. krohi sp. nov., which again mirrors the assumed phylogenetic relationships.

\section{Conclusion}

All four species in Breviturma subgen. nov. are similar to each other in some characters. In granule density and in the presence/absence of annulations on the arm spines, O. krohi sp. nov. seems to be related to $O$. doederleini. The higher number of arm spines separates $O$. brevipes from the other three species. Ophiocoma dentata and O. doederleini share several colour morphs. Ophiocoma krohi sp. nov. is the smallest of these species with about $16 \mathrm{~mm}$ dd, a difficult character that only clearly distinguishes significantly larger specimens. It is currently impossible to deduce the ancient states of any characters or infer a phylogeny within the subgenus by morphology. However, a comprehensive phylogenetic analysis of the whole genus may offer better clues to solving this problem.

The identity of the new species was difficult to reveal, since several nominal species have been synonymized with $O$. dentata and each one could potentially be conspecific with the new species. The spine number and granule density appear to be the most important characters to differentiate these species. However, the ophiuroid skeleton grows continuously and the number, shape and proportions of various parts are known to change during growth (Stöhr 2005). For instance, we show that in Ophiocoma krohi sp. nov. the granule density decreases with size. This complicates comparisons with type material that is naturally only available at limited size ranges and usually not available for dissection.

The recent description of two new species (Benavides-Serrato \& O'Hara 2008 and this study) in a relatively easily accessible (shallow water), conspicuous and well-studied group (ophiocomid brittle stars) suggests that a considerable part of marine biodiversity may remain to be discovered.

\section{Acknowledgements}

We are very grateful to A. Kroh, Vienna, for photographing and measuring the specimens of O. marmorata, and counting their spines. We are greatly indebted to G. Paulay and J. Slapcinsky for access to the FLMNH collection and for contributing specimens and images. Our deepest gratitude to H. Bruggemann who provided all specimens of $O$. krohi used in the morphological examinations (now type material) and the comparative material from the UR collections, and graciously allowed dissection and distribution of types to different institutions. Many thanks also to A. Baldinger, Harvard, for taking photos of the syntypes of $O$. insularia, and to C. Lüter, Berlin, for providing access to the types of 
STÖHR S. et al., Taxonomy and phylogeny of the Ophiocoma brevipes group

$O$. brevipes, $O$. dentata and $O$. ternispina. Funding for collection of specimens was provided by the French Agence Nationale de la Recherche (program BIOTAS, no. ANR-06-BDIV-002) and the National Science Foundation (NSF DEB-0529724). We thank two anonymous referees for thoughtful comments that improved the manuscript.

\section{References}

Benavides-Serrato M. \& O'Hara T.D. 2008. A new species in the Ophiocoma erinaceus complex from the South-west Pacific Ocean (Echinodermata: Ophiuroidea: Ophiocomidae). Memoirs of Museum Victoria 65: 51-56.

Clark H.L. 1915. Catalogue of recent Ophiurans: based on the collection of the Museum of Comparative Zoology. Memoirs of the Museum of Comparative Zoology 25 (4): 163-376.

Clark H.L. 1921. The echinoderm fauna of Torres Strait: its composition and its origin. Papers from the Department of Marine Biology of the Carnegie Institution of Washington 10, Carnegie Institution of Washington, Washington. http://dx.doi.org/10.5962/bhl.title.14613

Devaney D.M. 1970. Studies on ophiocomid brittlestars. I. A new genus (Clarkcoma) of Ophiocominae with a reevaluation of the genus Ophiocoma. Smithsonian Contributions to Zoology 51, Smithsonian Institution Press, Washington. http://dx.doi.org/10.5479/si.00810282.51

Farquhar H. 1897. A contribution to the history of N.Z. Echinoderms. Journal of the Linnean Society 26 (167): 186-198. http://dx.doi.org/10.1111/j.1096-3642.1897.tb00402.x

Hoareau T.B. \& Boissin E. 2010. Design of phylum-specific hybrid primers for DNA barcoding: addressing the need for efficient COI amplification in the Echinodermata. Molecular Ecology Resources 10 (6): 960-967. http://dx.doi.org/10.1111/j.1755-0998.2010.02848.x

Hoareau T.B., Boissin E., Paulay G. \& Bruggemann J.H. 2013. The Southwestern Indian Ocean as a potential marine evolutionary hotspot: perspectives from comparative phylogeography of reef brittlestars. Journal of Biogeography 40 (11): 2167-2197. http://dx.doi.org/10.1111/jbi.12155

Loriol P. de 1899. Notes pour servir a l'histoire des Echinodermes. Mémoires de la Société de Physique et d'Histoire Naturelle de Genève 33 (2): 1-34.

Lyman T. 1862. Descriptions of new Ophiuridae. Proceedings of the Boston Society of Natural History 8: $75-86$.

Marktanner-Turneretscher G. 1887. Beschreibung neuer Ophiuriden und Bemerkungen zu bekannten. Annalen des k.k. naturhistorischen Hofmuseums 2, A. Hölder, Wien. http://dx.doi.org/10.5962/bhl. $\underline{\text { title. } 11717}$

Martens E. von 1870. Die Ophiuriden des indischen Oceans. Archiv für Naturgeschichte 36: 244-262.

Müller J.H. \& Troschel F.H. 1842. System der Asteriden. F. Vieweg \& Sohn, Braunschweig.

O'Hara T.D., Byrne M. \& Cisternas P.A. 2004. The Ophiocoma erinaceus complex: another case of cryptic speciation in echinoderms. In: Heinzeller T. \& Nebelsick J.H. (eds) Echinoderms: München. Proceedings of the 11th International Echinoderm Conference: 537-542. Balkema, Leiden.

Palumbi S.R. 1996. Nucleic acids II: the polymerase chain reaction. In: Hillis D.M., Moritz C. \& Mable B.K. (eds) Molecular Systematics: 205-247. Sinauer Associates, Sunderland, Mass.

Peters W. 1851. Übersicht der an der Küste von Mossambique eingesammelten Ophiuren, unter denen sich zwei neue Gattungen befinden. Bericht über die zur Bekanntmachung geeigneten Verhandlungen der Königlich Preussischen Akademie der Wissenschaften zu Berlin 1851: 463-466. 
Price A.R.G. \& Rowe F.W.E. 1996. Indian Ocean echinoderms collected during the Sindbad Voyage (1980-81): 3. Ophiuroidea and Echinoidea. Bulletin of the Natural History Museum London (Zoology) 62 (2): 71-82.

Rasband W.S. 1997-2012. ImageJ. U. S. National Institutes of Health, Bethesda, Maryland. http:// imagej.nih.gov/ij/

Rowe F.W.E. \& Pawson D.L. 1977. A catalogue of echinoderm type-specimens in the Australian Museum, Sydney. Records of the Australian Museum 30 (14): 337-364. http://dx.doi.org/10.3853 j.0067-1975.30.1977.392

Smith E.A. 1876. Descriptions of two new species of Ophiocoma. Annals and Magazine of Natural History, series 4 18: 39-40.

Stöhr S. 2005. Who's who among baby brittle stars (Echinodermata: Ophiuroidea): postmetamorphic development of some North Atlantic forms. Zoological Journal of the Linnean Society 143 (4): 543-576. http://dx.doi.org/10.1111/j.1096-3642.2005.00155.x

Stöhr S., Conand C. \& Boissin E. 2008. Brittle stars (Echinodermata: Ophiuroidea) from La Réunion and the systematic position of Ophiocanops Koehler, 1922. Zoological Journal of the Linnean Society 153 (3): 545-560. http://dx.doi.org/10.1111/j.1096-3642.2008.00401.x

Stöhr S. \& O’Hara T.D. 2013. World Ophiuroidea Database. Vlaams Instituut voor de Zee. Available from http://www.marinespecies.org/ophiuroidea [accessed 5 Jul. 2013].

Stöhr S., O’Hara T.D. \& Thuy B. 2012. Global diversity of brittle stars (Echinodermata: Ophiuroidea). PLOS ONE 7 (3): 1-14. http://dx.doi.org/10.1371/journal.pone.0031940

Tamura K., Peterson D. \& Peterson N. 2011. MEGA5: Molecular Evolutionary Genetics Analysis using Maximum Likelihood, Evolutionary Distance, and Maximum Parsimony Methods. Molecular Biology and Evolution 28 (10) : 2731-2739. http://dx.doi.org/10.1093/molbev/msr121

Manuscript received: 8 July 2013

Manuscript accepted: 26 September 2013

Published on: 9 December 2013

Topic editor: Rudy Jocqué

Desk editor: Charlotte Thionois

Printed versions of all papers are also deposited in the libraries of the institutes that are members of the EJT consortium: Muséum National d'Histoire Naturelle, Paris, France; National Botanic Garden of Belgium, Meise, Belgium; Royal Museum for Central Africa, Tervuren, Belgium; Natural History Museum, London, United Kingdom; Royal Belgian Institute of Natural Sciences, Brussels, Belgium; Natural History Museum of Denmark, Copenhagen, Denmark. 